\title{
Palladium-catalysed reactions of conjugated enyne oxiranes with organoborons: A diastereoselective method of the synthesis of 2,4,5- trienol derivatives
}

\author{
Fırat Ziyanak ${ }^{\mathrm{a}}$, Melih Kuş ${ }^{\mathrm{a}}$, Leman Alkan-Karadeniz ${ }^{\mathrm{b}}$, Levent Artok ${ }^{\mathrm{a}, *}$ \\ a Department of Chemistry, Faculty of Science, Izmir Institute of Technology, Urla, Izmir 35430, Turkey \\ ${ }^{\mathrm{b}}$ Department of Chemistry, Faculty of Science, Ege University, Bornova, Izmir 35040, Turkey
}

\section{A R T I C L E I N F O}

\section{Article history:}

Received 12 September 2017

Received in revised form

26 January 2018

Accepted 11 May 2018

Available online 12 May 2018

\begin{abstract}
A B S T R A C T
A palladium-catalysed reaction of conjugated enyne oxiranes with organoboron reagents is described. This method allows aryl-substituted vinylallenes containing a hydroxyl group on the allylic position to be synthesized, with good diastereomeric ratios, under mild conditions.
\end{abstract}

() 2018 Elsevier Ltd. All rights reserved.

\section{Keywords:}

Allene

Vinylallene

2,4,5-Trienol

Enyne oxirane

Organoboron

\section{Introduction}

Allenes are highly versatile reagents, with broad utility as building blocks to a diverse array of high-value products. ${ }^{1}$ In addition, there are quite a number of biologically active natural products and pharmaceutical agents that contain an allene moiety. ${ }^{1 a, b}$ Therefore it is of interest to develop mild and selective methods to synthesise allenes with diverse substitution patterns ${ }^{2,3}$ that can be elaborated into specific targets. ${ }^{1,4}$

Our group has recently developed general palladium-catalysed methods to synthesise functionalized tetra-substituted allenes in a series of studies of conjugated enyne compounds. ${ }^{5}$ This work was initiated with studies of enynes containing a carbonate-leaving group on the allylic carbon. These enynes react with $\mathrm{CO} / \mathrm{ROH}$ combinations or organoboron reagents to afford esters and aryl- or alkenyl-substituted vinylallenes, respectively (Scheme 1 ). ${ }^{5 \mathrm{a}-\mathrm{c}}$

We then extended the alkoxycarbonylation to conjugated enynes carrying an oxirane moiety to synthesise functionalized vinylallenes, thereby allowing the formation of 7-hydroxy-2,3,5trienoates stereoselectively (Scheme 1). ${ }^{5 \mathrm{~d}, 6}$

\footnotetext{
* Corresponding author.

E-mail address: leventartok@iyte.edu.tr (L. Artok).
}

We demonstrate in this study that enyne oxiranes are compliant reagents for Miyaura-Suzuki-type reactions ${ }^{7}$ and thus produce aryl- or alkenyl-substituted vinylallenes bearing a hydroxyl group on the allylic position (Scheme 2).

\section{Results and discussion}

A promising result was obtained in the reaction of the enyne oxirane 1a, which had a dimethyl group on the oxirane terminus, with phenylboronic acid in the presence of $\mathrm{Pd}\left(\mathrm{PPh}_{3}\right)_{4}(3 \mathrm{~mol} \%)$ in a $\mathrm{THF} /$ water $(2: 1)$ mixture at $50^{\circ} \mathrm{C}$. This reaction resulted in complete conversion in $1.5 \mathrm{~h}$ and furnished the phenyl-substituted vinylallene 3aa, which had a hydroxyl group on the allylic position (Scheme 3).

Based on this promising result, the optimization studies with the enyne oxirane $\mathbf{1 b}$, which had a disubstituted oxirane ring, were carried out so that the stereoselectivity of the process could be evaluated with respect to the relative configurations of the axiallychiral allenyl moiety and the hydroxyl-substituted chirality centre (Scheme 4). However, interestingly, the reaction of this substrate with phenylboronic acid under the aforementioned conditions led only to a condensation product. Other organoboron reagents, such as $\mathrm{PhBF}_{3} \mathrm{~K}$ and $(\mathrm{PhBO})_{3}$ also shared the same fate. 

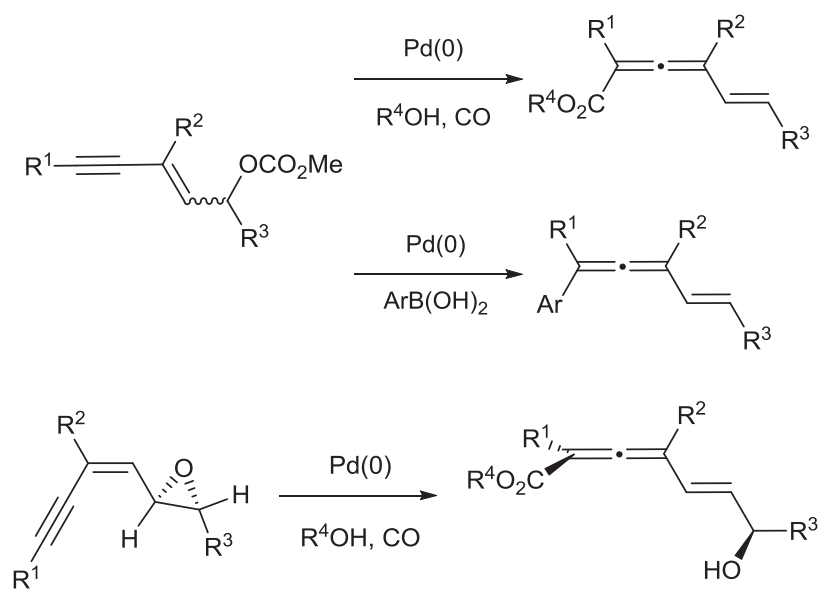

Scheme 1. Palladium-catalysed alkoxycarbonylation and Miyaura-Suzuki type reactions of enyne carbonates and enyne oxiranes. ${ }^{5}$

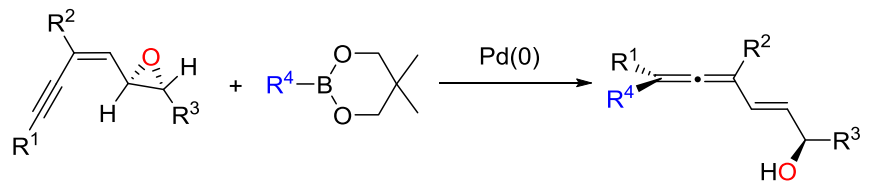

Scheme 2. This study: Palladium-catalysed arylation of enyne oxiranes.

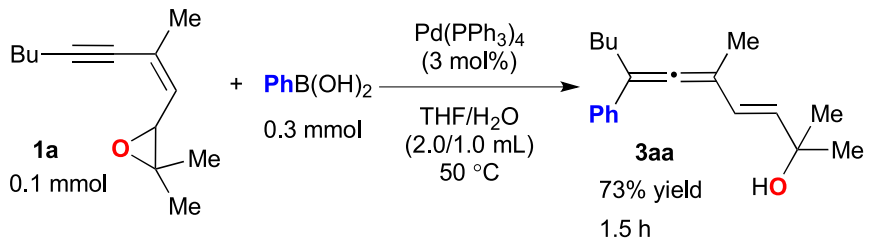

Scheme 3. Palladium-catalysed reaction of $\mathbf{1 a}$ and phenylboronic acid.

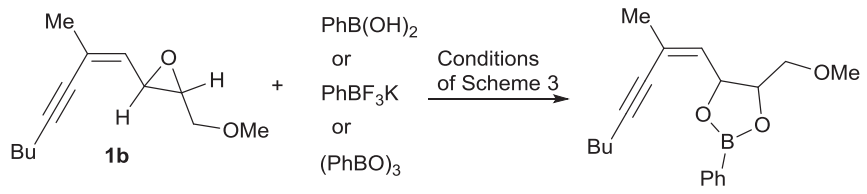

Scheme 4. Palladium-catalysed reaction of $\mathbf{1 b}$ and organoborons.

Palladium-catalysed condensation of allylic oxiranes with organoboronic acids has been previously documented, ${ }^{8}$ so the latter two reagents were probably also partially hydrolysed to phenylboronic acid under aqueous conditions. Encouragingly, the desired vinylallene product 3 ba could be obtained with a moderate yield (67\%) and diastereomeric ratio ( $\mathrm{dr}$ ) of 79:21, as determined by ${ }^{1} \mathrm{H}$ NMR, with the use of the $\mathrm{NaBPh}_{4}$ reagent (Table 1, entry 1 ). Increasing the $\mathrm{THF} /$ water ratio improved the reaction efficiency and dr to some extent (entry 2). However, lowering the reaction temperature to $25^{\circ} \mathrm{C}$ resulted in a significant reduction in the yield and led to the formation of the allylic substitution product $\mathbf{4 b a}$ (entry 3 ). The neopentyl glycol ester of phenylboronic acid (2a) was also compatible-when reacting with $\mathbf{1 b}$ at $25^{\circ} \mathrm{C}$ in the presence of $\mathrm{Pd}\left(\mathrm{PPh}_{3}\right)_{4}$ or $\mathrm{Pd}_{2}(\mathrm{dba})_{3}-\mathrm{CHCl}_{3} / \mathrm{PPh}_{3} \quad(3 \mathrm{~mol} \% \mathrm{Pd} ; \mathrm{P} / \mathrm{Pd}=4.5: 1$ ) catalyst systems, neither the condensation by-product formation nor $\mathbf{4 b a}$ were observed to form and, thus, 3ba could be obtained with a good yield, but with a non-satisfactory dr (entries 4 and 5).
Several mono and bidentate ligands were surveyed in an attempt to improve the efficacy and stereoselectivity for the reaction of $\mathbf{1 b}$ and $\mathbf{2 a}$ (see the supporting file), among which the most promising ligands that provided dr levels greater than 80:20 are listed in Table 2.

The reactions in the presence of TFP, $\mathrm{PPh}_{2} \mathrm{Me}, \mathrm{AsPh}_{3}$, dppe, dppp, and dppb ligands were either unaffordably slow or had unsatisfactory yields (entries $1-6$ ). The best performance in terms of the 3ba yield and reaction rate was achieved by the use of DPEPhos ligand, albeit with somewhat lessened stereoselectivity (entry 7).

Gratifyingly, the presence of a base additive has been found to be beneficial for the level of stereoselectivity (see the supporting file) and the organic bases usually performed better than inorganic bases (entries $8-10$ ). The additive $(i-\mathrm{Pr})_{2} \mathrm{EtN}$ was determined to be the most suitable base and even better diastereoselectivities were achieved by using larger amounts of the base (entries 11 and 12).

Having completed the optimization study, we surveyed the scope of the method against an array of neopentyl glycol esters of arylboronic acids with a variety of substitution patterns under the conditions of entry 11 of Table 2, which was judged to be sufficient for the selective formation of the desired product 3 . The palladiumcatalysed reaction of $\mathbf{1 b}$ and organoboron reagents (2b-e) substituted with an electron donating group gave the expected vinylallenes in good yields and stereoselectivities in relatively short reaction periods, regardless of the substitution pattern of $\mathbf{2}$ (Table 3 , entries $1-4$ ). However, the allylic substitution product $\mathbf{4 b f}$ was the main product of the reaction carried out with the highly encumbered 2,6-dimethyl substituted organoboron $\mathbf{2 f}$, and therefore, yielded the desired $\mathbf{3 b f}$ in low yield (entry 5).

Both electron-poor organoboron reagents (entries 6-8) and naphthylborons (entries 9 and 10) were competent reagents in terms of process efficiency and stereoselective formation of respective products. The reactions proceeded slower when the former organoboron reagents were used. The method was less stereoselective for alkenylboron reagents, whereas the structurally interesting divinylallene product $\mathbf{3 b l}$ could be isolated with a synthetically useful $\mathrm{dr}$ (83:17) when using (E)-1-pentenylboronic ester (entry 11 ). On the other hand, the reaction with $(E)$-styrylboronic ester proceeded with remarkably low diastereomeric selectivity (entry 12). Interestingly, a $\beta$-hydride elimination product was the sole product of the reaction performed in the presence of 3thienylboron (2n) (entry 13).<smiles>C=C(C#CBr)/C=C/C(O)COC</smiles>

The enyne oxirane component of the reaction was also varied, usually with similar success (Table 4). The substrate 1a showed a better performance under the optimized conditions. Hence, 3aa could be obtained in high yield (entry 1 ). The method was also suitable to the synthesis of the vinylallene with a primary allylic alcohol portion (3ca), although it was obtained in a relatively lower yield (entry 2). While the pendant oxygen functionality within $\mathrm{R}^{3}$ group could also be used in benzyl- or silyl-protected forms effectively, the protection of the hydroxyl functionality was not compulsory, although there was some reduction in yield without such protection (entries 3-5). The presence of a larger group in $\mathrm{R}^{3}$, such as 2-methoxypropan-2-yl group (19), was also well-tolerated by the method (entry 6 ). However, the reaction was dramatically decelerated when there was only one methyl group at the oxirane terminus (1h) (entry 7). 
Table 1

Effect of Organoboron type and other reaction parameters. ${ }^{a}$

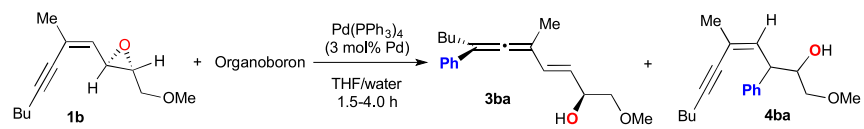

\begin{tabular}{|c|c|c|c|c|c|}
\hline Entry & Organo-boron & THF/water $(\mathrm{mL})$ & Temp. $\left({ }^{\circ} \mathrm{C}\right)$ & Yield $(\%)^{\mathrm{b}}$ 3ba:4ba & $\mathrm{dr}$ of $\mathbf{3} \mathbf{b a}^{\mathrm{b}}$ \\
\hline 1 & $\mathrm{NaBPh}_{4}$ & $2: 1$ & 50 & 67:- & $79: 21$ \\
\hline 2 & $\mathrm{NaBPh}_{4}$ & $2: 0.5$ & 50 & 75:- & $83: 17$ \\
\hline 3 & $\mathrm{NaBPh}_{4}$ & $2: 0.5$ & 25 & $31: 33$ & N.D. \\
\hline 4 & PhNeop & $2: 0.5$ & 25 & 77:- & $73: 27$ \\
\hline $5^{c}$ & PhNeop & $2: 0.5$ & 25 & 81:- & $76: 24$ \\
\hline
\end{tabular}

${ }^{a}$ Reagents and conditions: $\mathbf{1 b}(0.1 \mathrm{mmol})$, organoboron $(0.3 \mathrm{mmol})$.

b Determined by ${ }^{1} \mathrm{H}$ NMR using $p$-anisaldehyde as the internal standard.

c Performed using $\mathrm{Pd}_{2}(\mathrm{dba})_{3}-\mathrm{CHCl}_{3} / \mathrm{PPh}_{3}$ (3 mol\% $\mathrm{Pd}, \mathrm{P} / \mathrm{Pd}=4.5: 1$ ) combination.

Table 2

Optimization studies. ${ }^{\text {a }}$

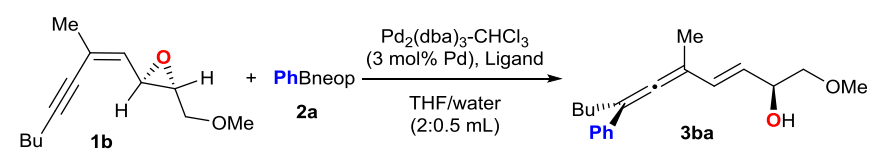

\begin{tabular}{llllll}
\hline Entry & Ligand $^{\mathrm{b}}$ & Base (eq) & Time $(\mathrm{h})$ & ${\text { Yield }(\%)^{\mathrm{c}}}$ & $\mathrm{dr}^{\mathrm{c}}$ \\
\hline 1 & $\mathrm{TFP}$ & - & 15 & 65 & $85: 15$ \\
2 & $\mathrm{PPh}_{2} \mathrm{Me}$ & - & 23 & 70 & $85: 15$ \\
3 & $\mathrm{AsPh}_{3}$ & - & 20 & 66 & $82: 18$ \\
4 & dppe & - & 30 & 81 & $84: 16$ \\
5 & dppp & - & 72 & 70 & $82: 18$ \\
6 & dppb & - & 40 & 48 & $83: 17$ \\
7 & DPEPhos & - & 1.5 & 92 & $80: 20$ \\
8 & DPEPhos & $\mathrm{NaHCO}_{3}(2)$ & 3.5 & 96 & $87: 13$ \\
9 & DPEPhos & $\mathrm{Et}_{3} \mathrm{~N}(2)$ & 5 & 90 & $89: 11$ \\
10 & DPEPhos & $(i-\operatorname{Pr})_{2}$ EtN $(2)$ & 2 & 90 & $90: 10$ \\
11 & DPEPhos & $(i-\operatorname{Pr})_{2}$ EtN $(4)$ & 4 & $92^{\mathrm{d}}$ & $91: 9$ \\
12 & DPEPhos & $(i \text {-Pr })_{2}$ EtN $(6)$ & 6 & 89 & $92: 8$ \\
\hline
\end{tabular}

a Reagents and conditions: $\mathbf{1 b}(0.1 \mathrm{mmol}), \mathbf{2 a}(0.3 \mathrm{mmol})$ at $25^{\circ} \mathrm{C}$.

b $\mathrm{P}$ (or As)/Pd: 4.5:1; TFP: tri(2-furyl)phosphine; dppe: 1,2-Bis(di-phenylphosphino)-ethane; dppp: 1,3-Bis(diphenylphosphino)propane; dppb: 1,4-Bis(diphenylphosphino)butane; DPEPhos: Bis[(2-diphe-nylphosphino)-phenyl] ether.

${ }^{c}$ Determined by ${ }^{1} \mathrm{H}$ NMR using $p$-anisaldehyde as the internal standard.

d Yield of isolated product is provided.

The reactions proceeded smoothly with enyne oxiranes bearing a terminal alkyne moiety (1i) and those bearing methyl (1j), phenyl $(\mathbf{1 k})$, or ester (11) groups on the alkynyl terminus $\left(R^{1}\right)$ (entries 8-11). However, the regioselectivity of the method was sensitive to the size of the $\mathrm{R}^{1}$ and $\mathrm{R}^{2}$ group on the alkenyl carbon that was in the vicinity of the alkynyl moiety (entries 12-17). When either group was larger than $\mathrm{Bu}$, the formation of the desired vinylallene products was accompanied by the corresponding allylic substitution products 4 , with varying yields of $9-16 \%$ for enynes where $R^{1}$ was Cy $(\mathbf{1 m})$ or $t-B u(\mathbf{1 n})$ or $R^{2}$ was Cy (1q) under the standard conditions. However, the formation of these by-products could be minimized by using $\mathrm{Ph}_{3} \mathrm{P}$, albeit with a decrease of $\mathrm{dr}$ of the product. On the other hand, the formation of 4ra was almost exclusive when $\mathrm{R}^{2}$ was $t$-Bu and the DPEphos ligand was used, and 3ra formation was not satisfactory even using the $\mathrm{Ph}_{3} \mathrm{P}$ ligand (entry 17).

The diastereomeric forms of the products $\mathbf{3}$ were estimated by comparison with NMR spectra of 3la (entry 11) and its diastereomeric form 3la' synthesized in another study ${ }^{5 \mathrm{~d}}$ (see the supporting information).
Table 3

The reaction of $\mathbf{1 b}$ with various organoboronic esters. ${ }^{a}$

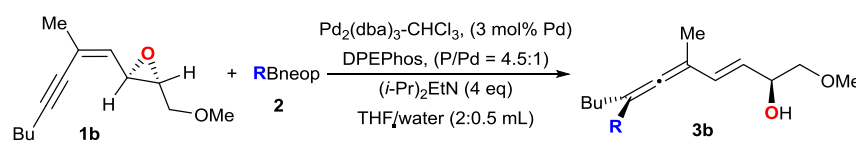

\begin{tabular}{|c|c|c|c|c|}
\hline Entry & $\mathrm{R}$ & Time (h) & Yield (\%) & $\mathrm{dr}^{\mathrm{b}}$ \\
\hline 1 & $p-\mathrm{MeC}_{6} \mathrm{H}_{4} \mathbf{2 b}$ & 1.5 & $93 \mathbf{3 b b}$ & $92: 8$ \\
\hline 2 & $m-\mathrm{MeC}_{6} \mathrm{H}_{4} \mathbf{2 c}$ & 2 & $72 \mathbf{3 b c}$ & $90: 10$ \\
\hline 3 & $o-\mathrm{MeC}_{6} \mathrm{H}_{4} \mathbf{2 d}$ & 1.5 & 87 3bd & $92: 8$ \\
\hline 4 & $p-\mathrm{OMeC}_{6} \mathrm{H}_{4} \mathbf{2 e}$ & 1.5 & 95 3be & $91: 9$ \\
\hline $5^{c}$ & $2,6-\mathrm{Me}_{2} \mathrm{C}_{6} \mathrm{H}_{4} \mathbf{2 f}$ & 20 & $28 \mathbf{3 b f}$ & $74: 26$ \\
\hline 6 & $p-\mathrm{CF}_{3} \mathrm{C}_{6} \mathrm{H}_{4} \mathbf{2 g}$ & 12 & 81 3bg & $92: 8$ \\
\hline 7 & $m-\mathrm{ClC}_{6} \mathrm{H}_{4} \mathbf{2 h}$ & 9 & $78 \mathbf{3 b h}$ & $90: 10$ \\
\hline 8 & $0-\mathrm{FC}_{6} \mathrm{H}_{4} \mathbf{2} \mathbf{i}$ & 6 & $83 \mathbf{3 b i}$ & $92: 8$ \\
\hline 9 & 1-Naphthyl $\mathbf{2 j}$ & 1.5 & $93 \mathbf{3 b j}$ & $91: 9$ \\
\hline 10 & 2-Naphthyl 2k & 2 & 88 3bk & $91: 9$ \\
\hline 11 & (E)-1-Pentenyl $\mathbf{2 l}$ & 4 & $63 \mathbf{3 b l}$ & $83: 17$ \\
\hline 12 & (E)-Styryl 2m & 6 & $73 \mathbf{3 b m}$ & $70: 30$ \\
\hline $13^{\mathrm{d}}$ & 3-Thienyl 2n & 3 & trace $\mathbf{3 b n}$ & - \\
\hline
\end{tabular}

a Reagents and conditions: $\mathbf{1 b}(0.1 \mathrm{mmol}), \mathbf{2}(0.3 \mathrm{mmol})$ at $25^{\circ} \mathrm{C}$.

b Determined by ${ }^{1} \mathrm{H}$ NMR.

c The reaction also yielded $43 \%$ of $\mathbf{4 b f}$.

d A $\beta$-hydride elimination product was the only product of this reaction.<smiles>COC[C@H](O)/C=C/C(C)=C=C(c1ccccc1)C(OC)OC</smiles>

The scope of the method was also evaluated for an enyne oxirane with an $(E)$-configured alkenyl moiety $((E)-\mathbf{1 b})$; the reaction of this reagent with 2a proceeded with low stereoselectivity and produced $\mathbf{3} \mathbf{b a}$ ', the diastereomer of $\mathbf{3 b a}$, with a 76:24 $\mathrm{dr}$ (Scheme 5).

Finally the method was also examined for substrate 1s, which had an endocyclic double bond. However, unlike its acyclic counterparts listed herein, its reaction with $\mathbf{2 a}$ under the standard conditions resulted in only a mixture of 3,5-dienone structure (5sa) and the typical allyl-substituted by-product 4sa; therefore the expected product of vinylallene 3sa could not be isolated (Scheme 6). This vinylallene 3sa would have been precursor to $\mathbf{5 s a}$, and after it was formed, it would have undergone successive isomerization and tautomarization steps to form $\mathbf{5 s a}$ products.

It has been shown in previous studies that vinylallenes can be 
Table 4

Arylation of enyne oxiranes.
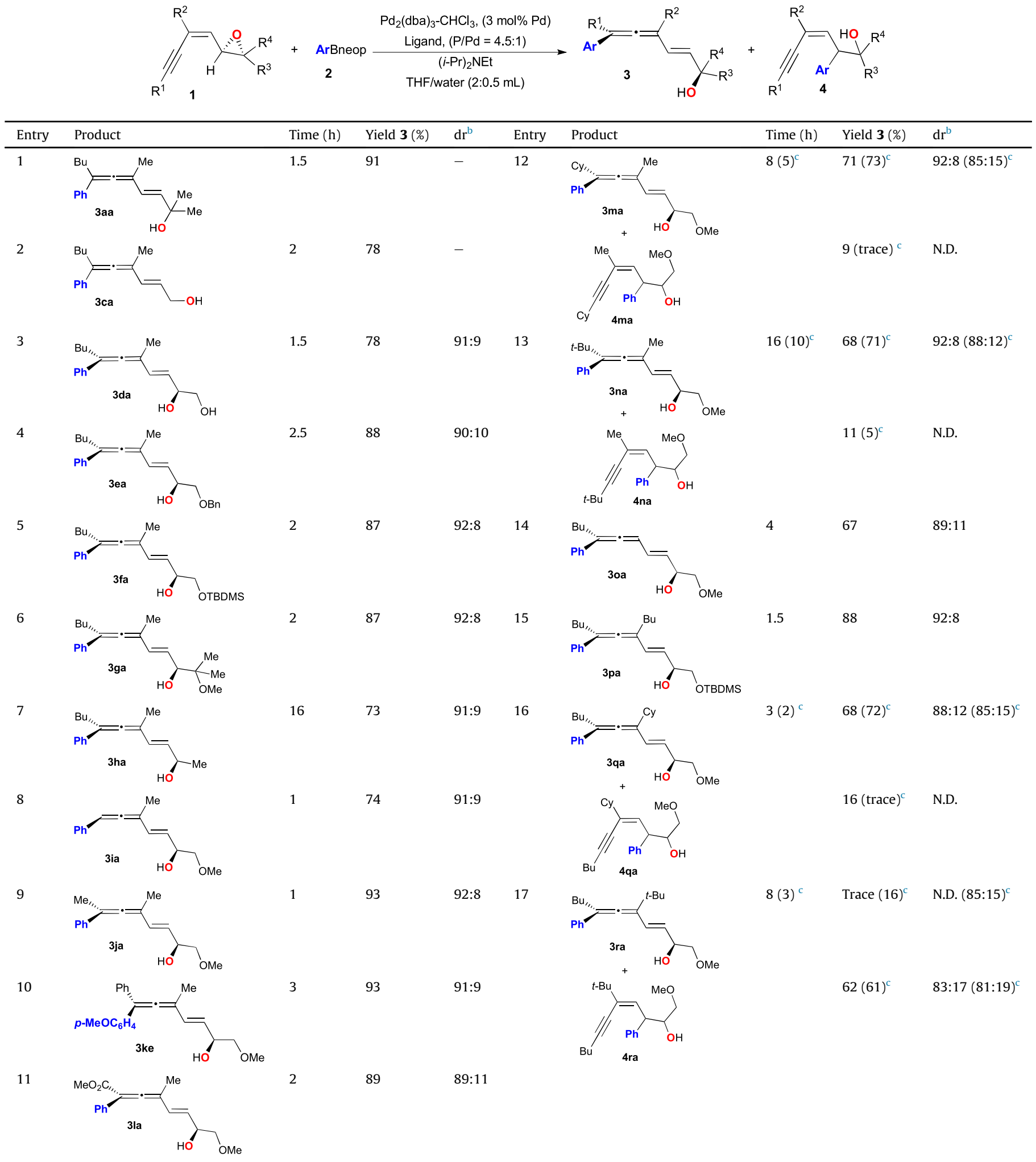

${ }^{\text {a }}$ Reagents and conditions: $\mathbf{1}(0.1 \mathrm{mmol}), \mathbf{2}(0.3 \mathrm{mmol})$, and DPEPhos ligand at $25^{\circ} \mathrm{C}$. The oxirane ring is in the form of $E$-configuration where it is applicable.

b Determined by ${ }^{1} \mathrm{H}$ NMR.

c $\mathrm{Ph}_{3} \mathrm{P}$ ligand was used in these reactions. 


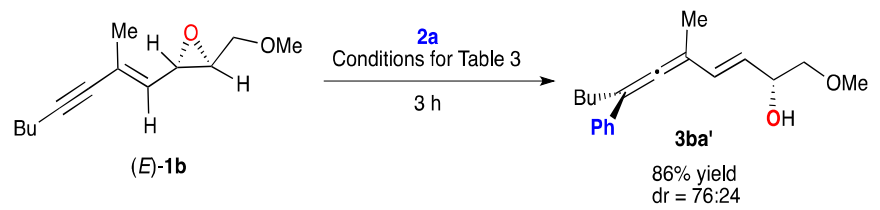

Scheme 5. The reaction of (E)-1b and $\mathbf{2 a}$.

converted to conjugated trienes via thermally induced [1,5] -sigmatropic hydrogen shift. ${ }^{9}$

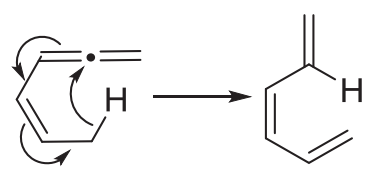

A hydrogen shift in this way requires that the alkenyl moiety of 3sa be present in the $(Z)$-configuration, but we do not know its exact configuration in fact. Whereas, in an our previous study, the palladium-catalysed alkoxycarbonylation of $1 \mathrm{~s}$ had yielded the corresponding ester functionalized vinylallene with exclusively $(E)$ configuration and no traces of a triene product could be detected in that study (Scheme 7). ${ }^{5 \mathrm{~d}}$

Considering that similar mechanisms are proposed for both alkoxycarbonylation $^{5 \mathrm{~d}}$ and arylation (this study, Scheme 8) reactions, we cannot envisage any possibility that could lead to the formation of 3sa in $(Z)$ configuration. Presumably both the locked scis conformational structure of 3sa and the apparent $\pi$-conjugated nature of the trienol intermediate may have favored an isomerization process.

As for the reaction mechanism, as suggested in our previous reports ${ }^{5}$ the catalytic cycle could begin with the formation of a $\pi$ allylpalladium intermediate in anti-form $(\mathbf{A})$, which has the $\mathrm{R}^{3}$ group oriented syn with respect to the middle allylic $\mathrm{C}-\mathrm{H}$ so that vinylallene products can ultimately form in (E)-configuration (Scheme 8). Then, this allyl-ligated palladium species $\mathbf{A}$ and organoboron 2 should undergo transmetalation to form the intermediate $\mathbf{B} .{ }^{10}$ The subsequent migration of the palladium unit over the intermediate $\mathbf{B}$ to the distal alkynyl carbon mainly by retention of the configuration, affords a $\sigma$-allenylpalladium complex $(\mathbf{C})^{11}$ and the final reductive elimination step then completes the cycle, resulting in the target product 3 . In cases where $\mathbf{B}$ has $R^{1}$ or $R^{2}$ groups relatively large in size or increased crowding around the palladium core metal, such a palladium shift would be less favourable and, therefore, $\mathbf{B}$ would preferentially undergo reductive elimination to furnish the by-product 4 .

The loss of stereochemical integrity of the resulting products

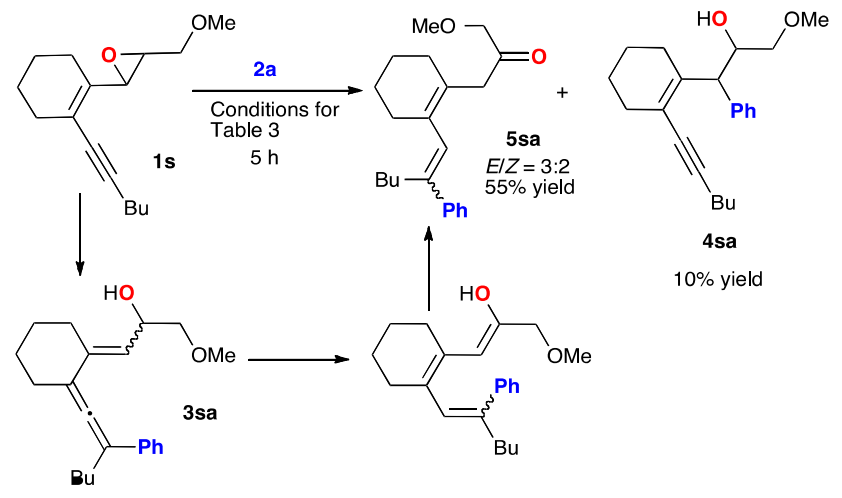

Scheme 6. Palladium-catalysed reaction of 1 s and 2a.<smiles>COCC(O)/C=C1\CCCC\C1=C\C(=O)OC</smiles>

Scheme 7. Pd-catalysed alkoxycarbonylation of $\mathbf{1 s} .^{5 d}$

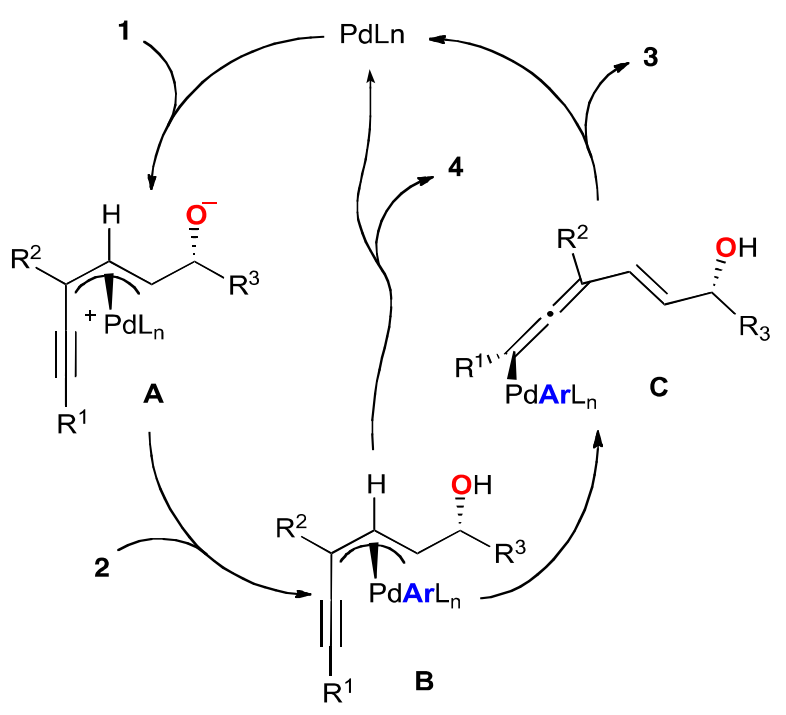

Scheme 8. Proposed mechanism.

probably took place during the course of the reaction cycle, because it was observed that the vinylallene $\mathbf{3 b a}$ was configurationally stable under the reaction conditions ${ }^{12}$; subjecting the purified $3 \mathbf{b a}$ again to the standard conditions for 3 days had no influence on its original diastereomeric ratio of $91: 9 .^{3 b, 5 c}$

Evidently, the vinylallene structures of this study are well-suited to Diels-Alder reactions, as the reaction of $\mathbf{3 b a}(\mathrm{dr}=91: 9)$ with $N$ phenylmaleimide proceeded with virtually complete facial- and endo-selectivity to furnish a structurally interesting adduct $\mathbf{6 b a}$ in two diastereomeric forms (91:9) (Scheme 9). The structure of the major diastereomeric form was elucidated by NOE studies.

\section{Conclusion}

We have demonstrated that the palladium-catalysed reactions of enyne oxiranes and organoboronic esters can be carried out with good stereoselectivity under mild conditions, yielding 2,4,5-trienol
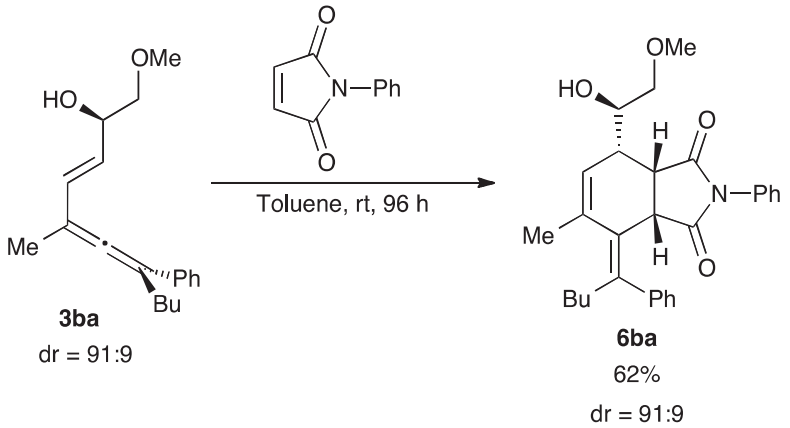

Scheme 9. The Diels-Alder reaction of the vinylallene 3ba with $N$-phenylmaleimide. 
derivatives. One product served as a substrate for a Diels-Alder reaction with high stereospecificity and stereoselectivity.

\section{Experimental section}

\subsection{General}

The synthesis procedure of the starting enyne oxirane compound $1 \mathbf{c}^{13}$ and others ${ }^{5 \mathrm{~d}}$ can be found elsewhere. The solvents were dried and purified following standard procedures. All of the reaction products were isolated by silica-gel column chromatography and analysed by GC-MS, NMR, FTIR, and HRMS techniques. NMR $(400 \mathrm{MHz})$ spectra were recorded in $\mathrm{CDCl}_{3}$ or $\mathrm{C}_{6} \mathrm{D}_{6}$. With $\mathrm{C}_{6} \mathrm{D}_{6}$ solvent, the ${ }^{1} \mathrm{H}$ NMR signals of diastereomers were usually resolved adequately, allowing determination of diastereomeric ratios ( $\mathrm{dr}$ ) smoothly. In contrast, when using $\mathrm{CDCl}_{3}$ solvent, diastereomeric signals were all overlapped. The coupling constants of olefinic protons and NOE studies confirmed (E)-configured structures for 3. Infrared spectra were obtained by the ATR method with neat samples. High-resolution mass spectral analyses of new compounds were performed using ESI-LTQ Orbitrap and an ESI-Q-TOF mass spectrometers.

\subsection{General method for the synthesis of neopentyl borates (2)}

The dry THF ( $1 \mathrm{~mL})$ solution of organoboronic acid (10 mmol), 2,2-dimethylpropan-1,3-diol (11 mmol, $1.2 \mathrm{~g}$ ), and $\mathrm{MgSO}_{4}$ (14 mmol, $1.7 \mathrm{~g}$ ) mixture was stirred overnight at room temperature under Ar. The crude mixture was concentrated under a reduced pressure and neopentyl borate ester derivative (2) was purified on a silica gel column with yields varying in the range of $76-97 \%$ (hexane/ethyl acetate as the eluent).

\subsection{General procedures for catalytic reactions}

A palladium complex, ligand, and the dry THF (half of the volume necessary for the reaction) were added successively into a Schlenk apparatus that is attached to an Ar line and stirred 15 min at $25^{\circ} \mathrm{C}$. Then, the dry THF (remaining half volume) solution of organoboron, enyne oxirane ( $\mathbf{1}$ ), and degassed water were added successively. The mixture was stirred magnetically in a preheated water or an oil bath. When the reaction was complete, as judged by TLC analysis, the solvent was evaporated under reduced atmosphere and the residue was purified by column chromatography on silica gel (hexane/ethyl acetate), affording the product $\mathbf{3}$ as colourless oil unless otherwise mentioned. In the case of optimization studies, the reaction mixture was filtered through a short silica gel column, washed with $\mathrm{Et}_{2} \mathrm{O}$, dried with $\mathrm{MgSO}_{4}$ and evaporated under reduced atmosphere. The residue was analysed by ${ }^{1} \mathrm{H}$ NMR using $p$-anisaldehyde as the internal standard. Aldehyde and methoxy hydrogen signals were used in the quantitative analyses.

\subsection{1. (E)-2,5-Dimethyl-7-phenylundeca-3,5,6-trien-2-ol (3aa)}

${ }^{1} \mathrm{H}$ NMR $\left(400 \mathrm{MHz}, \mathrm{CDCl}_{3}\right) \delta: 7.37-7.34(\mathrm{~m}, 2 \mathrm{H}), 7.33-7.38(\mathrm{~m}$ 2H), 7.17-7.21 (m, 1H), $6.23(\mathrm{~d}, J=15.9 \mathrm{~Hz}, 1 \mathrm{H}), 5.76(\mathrm{~d}, J=15.9 \mathrm{~Hz}$, $1 \mathrm{H}), 2.45(\mathrm{t}, J=8.0 \mathrm{~Hz}, 2 \mathrm{H}), 1.91(\mathrm{~s}, 3 \mathrm{H}), 1.54-1.39(\mathrm{~m}, 4 \mathrm{H}), 1.38(\mathrm{~S}$, $6 \mathrm{H}), 0.92(\mathrm{t}, J=8.0 \mathrm{~Hz}, 3 \mathrm{H}) ;{ }^{13} \mathrm{C}$ NMR $\left(100 \mathrm{MHz}, \mathrm{CDCl}_{3}\right) \delta: 208.1$, 137.4, 136.3, 128.5, 126.7, 126.3, 125.5, 105.3, 102.5, 71.2, 30.2, 30.1, 30.0, 22.6, 15.6, 14.2; FTIR $\left(v_{\max } / \mathrm{cm}^{-1}\right): 3356,2923,1609,1362$, 1142, 974, 763, 692; MS (EI, $m / z): 270\left(<5, \mathrm{M}^{+}\right), 252(6), 195(10)$, 165(12), 153(15), 141(40), 128(23), 115(35), 91(62), 77(50), 59(100); HRMS (ESI), $\mathrm{C}_{19} \mathrm{H}_{25}\left[\left(\mathrm{M}-\mathrm{H}_{2} \mathrm{O}\right)+\mathrm{H}\right]^{+}: 253.1951$ (calculated), 253.1952 (found).
4.3.2. ((2S,6S,E)-1-methoxy-5-methyl-7-phenylundeca-3,5,6-trien2-ol (3ba)

Isolated as a 91:9 mixture of diastereomers; white crystal; m.p. 46.4-47.8 ${ }^{\circ} \mathrm{C} ;{ }^{1} \mathrm{H}$ NMR $\left(400 \mathrm{MHz}, \mathrm{C}_{6} \mathrm{D}_{6}\right) \delta: 7.43$ (dd, $J=8.8,1.6 \mathrm{~Hz}$, $2 \mathrm{H}), 7.18(\mathrm{t}, J=7.2 \mathrm{~Hz}, 2 \mathrm{H}), 7.06(\mathrm{t}, J=8.0 \mathrm{~Hz}, 1 \mathrm{H}), 6.55(\mathrm{dd}, J=15.8$, $1.6 \mathrm{~Hz}, 1 \mathrm{H}$ ), 5.63 (dd, $J=15.8,5.9 \mathrm{~Hz}, 1 \mathrm{H}), 4.35-4.31$ (m, 1H), 3.14 (dd, A of ABX, $J_{A B}=9.6 \mathrm{~Hz}, J_{A X}=3.8 \mathrm{~Hz}, 1 \mathrm{H}$ ), 3.11 (dd, B of ABX, $\left.J_{A B}=9.6 \mathrm{~Hz}, J_{B X}=8.3 \mathrm{~Hz}, 1 \mathrm{H}\right), 3.01(\mathrm{~s}, 3 \mathrm{H}), 2.40(\mathrm{t}, J=7.2 \mathrm{~Hz}, 2 \mathrm{H})$, $1.86(\mathrm{~s}, 3 \mathrm{H}), 1.55-1.48(\mathrm{~m}, 2 \mathrm{H}), 1.37-1.28(\mathrm{~m}, 2 \mathrm{H}), 0.85(\mathrm{t}, J=7.6 \mathrm{~Hz}$, $3 \mathrm{H}) ;{ }^{13} \mathrm{C}$ NMR $\left(100 \mathrm{MHz}, \mathrm{C}_{6} \mathrm{D}_{6}\right) \delta: 208.8,138.0,130.4,129.1,128.6$, 127.3, 127.1, 106.3, 103.4, 77.5, 71.7, 59.0, 30.8, 30.7, 23.1, 15.9, 14.5; FTIR $\left(v_{\max } / \mathrm{cm}^{-1}\right): 3426,2938,1923,1455,1198,1126,968,770,697$; MS (EI, $m / z)$ : 286(8, $\left.\mathrm{M}^{+}\right), 236(8), 198(15), 169(40), 129(45), 115(52)$, 91(100), 77(88), 56(84); HRMS (ESI) $\mathrm{C}_{19} \mathrm{H}_{27} \mathrm{O}_{2}[\mathrm{M}+\mathrm{H}]^{+}: 287.2006$ (calculated); 287.2008 (found).

\subsection{3. (2S,6R,E)-1-methoxy-5-methyl-7-phenylundeca-3,5,6-trien- 2-ol (3ba')}

Isolated as a 76:24 mixture of diastereomers; ${ }^{1} \mathrm{H}$ NMR (400 MHz, $\mathrm{C}_{6} \mathrm{D}_{6}$ ) $\delta: ~ 7.45-7.41(\mathrm{~m}, 2 \mathrm{H}), 7.20-7.15$ ( $\left.\mathrm{m}, 2 \mathrm{H}\right), 7.08-7.04$ $(\mathrm{m}, 1 \mathrm{H}), 6.55(\mathrm{dd}, J=15.8,1.4 \mathrm{~Hz}, 1 \mathrm{H}), 5.633(\mathrm{dd}, J=15.8,6.0 \mathrm{~Hz}, 1 \mathrm{H})$, 4.35-4.30 (m, 1H), 3.14 (dd, A of $\left.A B X, J_{A B}=9.4 \mathrm{~Hz}, J_{A X}=3.4 \mathrm{~Hz}, 1 \mathrm{H}\right)$, $3.11\left(\mathrm{dd}, \mathrm{B}\right.$ of $\left.\mathrm{ABX}, J_{A B}=9.4 \mathrm{~Hz}, J_{B X}=7.9 \mathrm{~Hz}, 1 \mathrm{H}\right), 3.01(\mathrm{~s}, 3 \mathrm{H}), 2.40(\mathrm{t}$, $J=7.2 \mathrm{~Hz}, 2 \mathrm{H}), 1.86(\mathrm{~s}, 3 \mathrm{H}), 1.57-1.48(\mathrm{~m}, 2 \mathrm{H}), 1.38-1.28(\mathrm{~m}, 2 \mathrm{H})$, $0.86(\mathrm{t}, J=7.6 \mathrm{~Hz}, 3 \mathrm{H}) ;{ }^{13} \mathrm{C}$ NMR $\left(101 \mathrm{MHz}, \mathrm{C}_{6} \mathrm{D}_{6}\right) \delta: 208.8,138.0$, 130.5, 129.06, 127.3, 127.1, 106.3, 103.4, 77.5, 71.7, 58.9, 30.81, 30.75, 23.2, 15.9, 14.5; FTIR $\left(v_{\max } / \mathrm{cm}^{-1}\right): 3428,2931,2163,1925,1438$, 1115, 973, 761, 692; MS (EI, $m / z): 286\left(<5, \mathrm{M}^{+}\right)$241(20), 223(25), 199(30), 181(45), 169(100), 91(70), 77(20), 45(50).

\subsection{4. (Z)-1-Methoxy-5-methyl-3-phenylundec-4-en-6-yn-2-ol (4ba)}

${ }^{1} \mathrm{H}$ NMR (400 MHz, $\left.\mathrm{CDCl}_{3}\right): \delta 7.34-7.24(\mathrm{~m}, 5 \mathrm{H}), 5.75(\mathrm{~d}$, $J=10.2 \mathrm{~Hz}, 1 \mathrm{H}), 4.09(\mathrm{t}, J=8.4 \mathrm{~Hz}, 1 \mathrm{H}), 3.95(\mathrm{t}, J=9.6 \mathrm{~Hz}, 1 \mathrm{H}), 3.54$ (dd, $J=9.8,2.7 \mathrm{~Hz}, 1 \mathrm{H}), 3.39-3.30(\mathrm{~m}, 4 \mathrm{H}), 2.38(\mathrm{t}, J=7.2 \mathrm{~Hz}, 2 \mathrm{H})$, $1.82(\mathrm{~d}, J=1.2 \mathrm{~Hz}, 3 \mathrm{H}), 1.55-1.44(\mathrm{~m}, 4 \mathrm{H}), 0.95(\mathrm{t}, J=7.2 \mathrm{~Hz}, 3 \mathrm{H}) ;{ }^{13} \mathrm{C}$ NMR $\left(100 \mathrm{MHz}, \mathrm{CDCl}_{3}\right): \delta 141.0,134.7,128.7,128.3,126.7,120.2$, $94.9,79.5,75.2,73.0,59.1,50.5,30.9,23.7,22.0,19.2,13.6$.

\subsection{5. (2S,6S,E)-1-methoxy-5-methyl-7-(p-tolyl)undeca-3,5,6-} trien-2-ol (3bb)

Isolated as a 92:8 mixture of diastereomers; ${ }^{1} \mathrm{H}$ NMR $(400 \mathrm{MHz}$, $\left.\mathrm{C}_{6} \mathrm{D}_{6}\right) \delta: 7.41(\mathrm{~d}, J=8.2 \mathrm{~Hz}, 2 \mathrm{H}), 7.05(\mathrm{~d}, J=7.8 \mathrm{~Hz}, 2 \mathrm{H}), 6.56(\mathrm{dd}$, $J=15.8,1.0 \mathrm{~Hz}, 1 \mathrm{H}), 5.63(\mathrm{dd}, J=15.8,6.1 \mathrm{~Hz}, 1 \mathrm{H}), 4.34-4.28(\mathrm{~m}$, $1 \mathrm{H}), 3.14\left(\mathrm{dd}, A\right.$ of $\left.A B X, J_{A B}=9.4 \mathrm{~Hz}, J_{A X}=8.2 \mathrm{~Hz}, 1 \mathrm{H}\right), 3.10(\mathrm{dd}, \mathrm{B}$ of $\left.\mathrm{ABX}, J_{A B}=9.4 \mathrm{~Hz}, J_{B X}=3.5 \mathrm{~Hz}, 1 \mathrm{H}\right), 3.00(\mathrm{~s}, 3 \mathrm{H}), 2.43(\mathrm{t}, J=8 \mathrm{~Hz}, 2 \mathrm{H})$, $2.14(\mathrm{~s}, 3 \mathrm{H}), 1.87(\mathrm{~s}, 3 \mathrm{H}), 1.54$ (quint, $J=7.2 \mathrm{~Hz}, 2 \mathrm{H}), 1.37-1.30$ (m, $2 \mathrm{H}), 0.86(\mathrm{t}, J=7.6 \mathrm{~Hz}, 3 \mathrm{H}) ;{ }^{13} \mathrm{C}$ NMR $\left(101 \mathrm{MHz}, \mathrm{C}_{6} \mathrm{D}_{6}\right) \delta: 208.6$, 136.8, 135.1, 130.7, 129.8, 128.3, 127.1, 106.2, 103.3, 77.5, 71.7, 58.9, 30.9, 30.6, 23.2, 21.4, 16.0, 14.5; FTIR $\left(v_{\max } / \mathrm{cm}^{-1}\right)$ : 3444, 2923, 1523, 1441, 1197, 1123, 961, 831, 603; MS (EI, $m / z): 300\left(15, \mathrm{M}^{+}\right), 282(5)$, 225(30), 193(30), 155(50), 141(30), 105(20), 91(15), 44(100); HRMS (ESI): $\mathrm{C}_{20} \mathrm{H}_{28} \mathrm{O}_{2} \mathrm{Na}[\mathrm{M}+\mathrm{Na}]^{+}: 323.1982$ (calculated), 323.1983 (found).

\subsection{6. (2S,6S,E)-1-methoxy-5-methyl-7-(m-tolyl)undeca-3,5,6-} trien-2-ol (3bc)

Isolated as a 90:10 mixture of diastereomers; ${ }^{1} \mathrm{H}$ NMR $(400 \mathrm{MHz}$, $\left.\mathrm{C}_{6} \mathrm{D}_{6}\right) \delta: 7.36(\mathrm{~s}, 1 \mathrm{H}), 7.31(\mathrm{~d}, J=7.8 \mathrm{~Hz}, 1 \mathrm{H}), 7.17-7.13(\mathrm{~m}, 1 \mathrm{H}), 6.92$ (dt, $J=7.4,0.8 \mathrm{~Hz}, 1 \mathrm{H}), 6.58(\mathrm{dd}, J=15.9,1.4 \mathrm{~Hz}, 1 \mathrm{H}), 5.63$ (dd, $J=15.9,5.6 \mathrm{~Hz}, 1 \mathrm{H}), 4.34-4.30(\mathrm{~m}, 1 \mathrm{H}), 3.13(\mathrm{dd}, \mathrm{A}$ of $\mathrm{ABX}$, $\left.J_{A B}=9.4 \mathrm{~Hz}, J_{A X}=7.8 \mathrm{~Hz}, 1 \mathrm{H}\right), 3.10\left(\mathrm{dd}, \mathrm{B}\right.$ of $\mathrm{ABX}, J_{A B}=9.4 \mathrm{~Hz}$, $\left.J_{B X}=3.6 \mathrm{~Hz}, 1 \mathrm{H}\right), 3.00(\mathrm{~s}, 3 \mathrm{H}), 2.44(\mathrm{t}, J=6.4 \mathrm{~Hz}, 2 \mathrm{H}), 2.27(\mathrm{bs}, 1 \mathrm{H})$, $2.14(\mathrm{~s}, 3 \mathrm{H}), 1.88(\mathrm{~s}, 3 \mathrm{H}), 1.59-1.51(\mathrm{~m}, 2 \mathrm{H}), 1.40-1.30(\mathrm{~m}, 2 \mathrm{H}), 0.86$ $(\mathrm{t}, J=7.2 \mathrm{~Hz}, 3 \mathrm{H}) ;{ }^{13} \mathrm{C}$ NMR $\left(101 \mathrm{MHz}, \mathrm{C}_{6} \mathrm{D}_{6}\right) \delta: 208.8,138.4,138.0$, 
130.6, 129.1, 128.5, 128.2, 127.8, 124.3, 106.4, 103.2, 77.5, 71.7, 58.9, 30.9, 30.8, 23.2, 21.9, 16.0, 14.5; FTIR $\left(v_{\max } / \mathrm{cm}^{-1}\right)$ : 3435, 2922, 1606, 1470, 1258, 1107, 956, 797, 699; MS (EI, $m / z): 300\left(5, \mathrm{M}^{+}\right), 237(15)$, 195(40), 183(85), 143(45), 105(100), 91(40), 77(25), 45(40); HRMS (ESI) $\mathrm{C}_{20} \mathrm{H}_{29} \mathrm{O}_{2}[\mathrm{M}+\mathrm{H}]^{+}: 301.2162$ (calculated), 301.2164 (found).

\subsection{7. (2S,6S,E)-1-methoxy-5-methyl-7-(o-tolyl)undeca-3,5,6- trien-2-ol (3bd)}

Isolated as a 92:8 mixture of diastereomers; ${ }^{1} \mathrm{H} \mathrm{NMR}(400 \mathrm{MHz}$, $\left.\mathrm{C}_{6} \mathrm{D}_{6}\right) \delta: 7.26(\mathrm{~d}, J=6.7 \mathrm{~Hz}, 1 \mathrm{H}), 7.11-7.04(\mathrm{~m}, 3 \mathrm{H}), 6.58(\mathrm{dd}, J=15.7$, $1.6 \mathrm{~Hz}, 1 \mathrm{H}), 5.54(\mathrm{dd}, J=15.7,5.9 \mathrm{~Hz}, 1 \mathrm{H}), 4.34-4.29(\mathrm{~m}, 1 \mathrm{H}), 3.13$ (dd, A of ABX, $J_{A B}=9.4 \mathrm{~Hz}, J_{A X}=7.7 \mathrm{~Hz}, 1 \mathrm{H}$ ), 3.09 (dd, B of ABX, $\left.J_{A B}=9.4 \mathrm{~Hz}, J_{B X}=3.7 \mathrm{~Hz}, 1 \mathrm{H}\right), 3.00(\mathrm{~s}, 3 \mathrm{H}), 2.35(\mathrm{~s}, 3 \mathrm{H}), 2.33$ (td, $J=7.2,2.8 \mathrm{~Hz}, 2 \mathrm{H}), 1.80(\mathrm{~s}, 3 \mathrm{H}), 1.50-1.42(\mathrm{~m}, 2 \mathrm{H}), 1.36-1.26(\mathrm{~m}$, $2 \mathrm{H}), 0.84(\mathrm{t}, J=7.2 \mathrm{~Hz}, 3 \mathrm{H}) ;{ }^{13} \mathrm{C}$ NMR $\left(101 \mathrm{MHz}, \mathrm{C}_{6} \mathrm{D}_{6}\right) \delta: 206.9$, 138.6, 136.3, 131.2, 130.9, 128.8, 127.9, 127.5, 126.6, 104.9, 100.5, 77.5, 71.6, 58.9, 34.7, 30.7, 23.1, 21.2, 16.0, 14.5; FTIR $\left(v_{\max } / \mathrm{cm}^{-1}\right): 3413$, 2922, 1449, 1190, 1142, 959, 756, 728; MS (EI, $m / z): 300\left(20, \mathrm{M}^{+}\right)$, 282(5), 225(30), 193(30), 155(50), 141(30), 105(20), 91(15), 44(100); HRMS (ESI) $\mathrm{C}_{20} \mathrm{H}_{28} \mathrm{O}_{2} \mathrm{Na}[\mathrm{M}+\mathrm{Na}]^{+}: 323.1982$ (calculated), 323.1984 (found).

\subsection{8. (2S,6S,E)-1-methoxy-7-(4-methoxyphenyl)-5-methylundeca-}

3,5,6-trien-2-ol (3be)

Isolated as a 91:9 mixture of diastereomers; ${ }^{1} \mathrm{H}$ NMR $(400 \mathrm{MHz}$, $\left.\mathrm{C}_{6} \mathrm{D}_{6}\right) \delta: 7.38(\mathrm{~d}, J=9.2 \mathrm{~Hz}, 2 \mathrm{H}), 6.82(\mathrm{~d}, J=8.4 \mathrm{~Hz}, 2 \mathrm{H}), 6.58(\mathrm{dd}$, $J=15.8,1.4 \mathrm{~Hz}, 1 \mathrm{H}), 5.64(\mathrm{dd}, J=15.7,5.9 \mathrm{~Hz}, 1 \mathrm{H}), 4.37-4.32(\mathrm{~m}, 1 \mathrm{H})$, $3.33(\mathrm{~s}, 3 \mathrm{H}), 3.16\left(\mathrm{dd}, \mathrm{A}\right.$ of $\left.\mathrm{ABX}, J_{A B}=9.4 \mathrm{~Hz}, J_{A X}=4.3 \mathrm{~Hz}, 1 \mathrm{H}\right), 3.12$ (dd, B of $\left.A B X, J_{A B}=9.4 \mathrm{~Hz}, J_{B X}=7.6 \mathrm{~Hz}, 1 \mathrm{H}\right), 3.015(\mathrm{~s}, 3 \mathrm{H}), 2.42(\mathrm{t}$, $J=6.8 \mathrm{~Hz}, 2 \mathrm{H}), 1.89(\mathrm{~s}, 3 \mathrm{H}), 1.60-1.51(\mathrm{~m}, 2 \mathrm{H}), 1.40-1.31(\mathrm{~m}, 2 \mathrm{H})$, $0.87(\mathrm{t}, J=7.2 \mathrm{~Hz}, 3 \mathrm{H}) ;{ }^{13} \mathrm{C}$ NMR $\left(101 \mathrm{MHz}, \mathrm{C}_{6} \mathrm{D}_{6}\right) \delta: 208.4,159.6$, 130.9, 130.1, 128.24, 128.19, 114.7, 105.9, 103.3, 77.5, 71.7, 59.0, 55.2, 31.0, 30.9, 23.2, 16.1, 14.5; FTIR $\left(v_{\max } / \mathrm{cm}^{-1}\right): 3444,2931,2867,1925$, 1613, 1512, 1454, 1247, 1174, 1120, 1041, 967, 835, 597; MS (EI, m/z): 316(10, $\left.\mathrm{M}^{+}\right), 259(80), 209(100)$, 171(95), 121(75), 44(90); HRMS (ESI) $\mathrm{C}_{20} \mathrm{H}_{29} \mathrm{O}_{3}[\mathrm{M}+\mathrm{H}]^{+}: 317.2111$ (calculated), 317.2112 (found).

\subsection{9. (2S,6S,E)-7-(2,6-dimethylphenyl)-1-methoxy-5-}

methylundeca-3,5,6-trien-2-ol (3bf)

Isolated as a 74:26 mixture of diastereomers; ${ }^{1} \mathrm{H}$ NMR $(400 \mathrm{MHz}$, $\left.\mathrm{C}_{6} \mathrm{D}_{6}\right) \delta$ : 7.06-6.96 (m, 3H), $6.65(\mathrm{~d}, J=15.8 \mathrm{~Hz}, 1 \mathrm{H}), 5.52(\mathrm{dd}, 15.8$, $5.9,1 \mathrm{H}), 4.35-4.31(\mathrm{~m}, 1 \mathrm{H}), 3.13\left(\mathrm{dd}, \mathrm{A}\right.$ of $\mathrm{ABX}, J_{A B}=9.2 \mathrm{~Hz}$, $\left.J_{A X}=7.8 \mathrm{~Hz}, 1 \mathrm{H}\right), 3.08\left(\mathrm{dd}, \mathrm{B}\right.$ of $\left.\mathrm{ABX}, J_{A B}=9.2 \mathrm{~Hz}, J_{B X}=3.5 \mathrm{~Hz}, 1 \mathrm{H}\right)$, $3.00(\mathrm{~s}, 3 \mathrm{H}), 2.34(\mathrm{~s}, 6 \mathrm{H}), 2.13-2.07(\mathrm{~m}, 2 \mathrm{H}), 1.77(\mathrm{~s}, 3 \mathrm{H}), 1.55-1.48$ $(\mathrm{m}, 2 \mathrm{H}), 1.37-1.26(\mathrm{~m}, 2 \mathrm{H}), 0.85(\mathrm{t}, J=7.2 \mathrm{~Hz}, 3 \mathrm{H}) ;{ }^{13} \mathrm{C}$ NMR $\left(101 \mathrm{MHz}, \mathrm{C}_{6} \mathrm{D}_{6}\right) \delta: 204.8,138.9,136.0,130.7,128.3,127.7,127.5$, 104.1, 100.8, 77.5, 71.6, 58.9, 34.1, 30.5, 23.3, 20.8, 15.7, 14.6; FTIR $\left(v_{\max } / \mathrm{cm}^{-1}\right): 3421,2944,1470,1386,1190,1129,956,760 ; \mathrm{MS}$ (EI, $\mathrm{m} /$ $z)$ : 314(50, $\left.\mathrm{M}^{+}\right), 269(60), 197(45), 157(30), 119(55), 45(100)$; HRMS (ESI) $\mathrm{C}_{21} \mathrm{H}_{31} \mathrm{O}_{2}[\mathrm{M}+\mathrm{H}]^{+}: 315.2319$ (calculated); 315.2320 (found).

\subsubsection{0. (Z)-3-(2,6-dimethylphenyl)-1-methoxy-5-methylundec-4- en-6-yn-2-ol (4bf)}

${ }^{1} \mathrm{H}$ NMR (400 MHz, $\left.\mathrm{C}_{6} \mathrm{D}_{6}\right) \delta$ : 7.05-6.96 (m, 3H), $6.35(\mathrm{~d}$, $J=8.0 \mathrm{~Hz}, 1 \mathrm{H}), 4.69(\mathrm{t}, J=8.0 \mathrm{~Hz}, 1 \mathrm{H}), 4.31-4.23(\mathrm{~m}, 1 \mathrm{H}), 3.20-3.16$ (dd, $J=9.4,2.8 \mathrm{~Hz}, 1 \mathrm{H}), 3.14-3.07(\mathrm{~m}, 1 \mathrm{H}), 2.92(\mathrm{~s}, 3 \mathrm{H}), 2.69-2.28$ (bs, 6H), 2.11 (t, J=6.8 Hz, 2H), $1.87(\mathrm{~s}, 3 \mathrm{H}), 1.40-1.18(\mathrm{~m}, 4 \mathrm{H}), 0.77$ $(\mathrm{t}, J=8.0 \mathrm{~Hz}, 3 \mathrm{H})$; FTIR $\left(v_{\max } / \mathrm{cm}^{-1}\right): 3451,2912,1460,1391,1122$, 767; ${ }^{13} \mathrm{C}$ NMR $\left(101 \mathrm{MHz}, \mathrm{C}_{6} \mathrm{D}_{6}\right) \delta: 139.5,137.1,126.9,120.8,95.2$, 81.4, 75.4, 73.1, 58.9, 46.7, 31.5, 24.5, 22.8 (bs), 22.6, 20.0, 14.1; ${ }^{1} \mathrm{H}$ $\operatorname{NMR}\left(400 \mathrm{MHz}, \mathrm{CD}_{3} \mathrm{Cl}\right) \delta: 7.02-6.97(\mathrm{~m}, 3 \mathrm{H}), 6.14(\mathrm{~d}, J=8.0 \mathrm{~Hz}, 1 \mathrm{H})$, $4.43(\mathrm{t}, J=8.0 \mathrm{~Hz}, 1 \mathrm{H}), 4.29-4.22(\mathrm{~m}, 1 \mathrm{H}), 3.28(\mathrm{~s}, 3 \mathrm{H}), 3.28-3.22$ $(\mathrm{m}, 1 \mathrm{H}), 3.14-3.09(\mathrm{~m}, 1 \mathrm{H}), 2.43(\mathrm{~s}, 6 \mathrm{H}), 2.29(\mathrm{t}, J=8.0 \mathrm{~Hz}, 2 \mathrm{H}), 1.86$ $(\mathrm{s}, 3 \mathrm{H}), 1.51-1.33(\mathrm{~m}, 4 \mathrm{H}), 0.90(\mathrm{t}, J=8.0 \mathrm{~Hz}, 3 \mathrm{H}) ;{ }^{13} \mathrm{C} \mathrm{NMR}$ $\left(101 \mathrm{MHz}, \mathrm{CD}_{3} \mathrm{Cl}\right) \delta: 138.0,135.0,126.5,121.3,95.1,80.2,74.7,72.5$,
59.1, 46.2, 30.9, 24.2, 22.3 (bs), 22.2, 19.5, 13.8; (EI, $\mathrm{m} / \mathrm{z}): 314(<5$, $\mathrm{M}^{+}$), 269(15), 239(100), 198(55), 183(85), 169(95), 119(40), 55(40); HRMS (ESI) $\mathrm{C}_{21} \mathrm{H}_{30} \mathrm{O}_{2}[\mathrm{M}+\mathrm{H}]^{+}: 315.2319$ (calculated); 315.2320 (found).

4.3.11. (2S,6S,E)-1-methoxy-5-methyl-7-(4-(trifluoromethyl) phenyl)undeca-3,5,6-trien-2-ol (3bg)

Isolated as a 92:8 mixture of diastereomers; ${ }^{1} \mathrm{H}$ NMR $(400 \mathrm{MHz}$, $\left.\mathrm{C}_{6} \mathrm{D}_{6}\right) \delta: 7.37(\mathrm{~d}, J=8.2 \mathrm{~Hz}, 2 \mathrm{H}), 7.22(\mathrm{~d}, J=7.8 \mathrm{~Hz}, 2 \mathrm{H}), 6.54(\mathrm{dd}$, $J=15.7,1.6 \mathrm{~Hz}, 1 \mathrm{H}), 5.63(\mathrm{dd}, J=15.7,5.5 \mathrm{~Hz}, 1 \mathrm{H}), 4.32(\mathrm{dt}, J=3.8$, $1.8 \mathrm{~Hz}, 1 \mathrm{H}$ ), 3.15 (dd, A of ABX, $\left.J_{A B}=11.8 \mathrm{~Hz}, J_{A X}=9.6 \mathrm{~Hz}, 1 \mathrm{H}\right), 3.09$ (dd, B of $\left.A B X, J_{A B}=11.8 \mathrm{~Hz}, J_{B X}=2.1 \mathrm{~Hz}, 1 \mathrm{H}\right), 3.00(\mathrm{~s}, 3 \mathrm{H}), 2.24(\mathrm{t}$, $J=7.2 \mathrm{~Hz}, 2 \mathrm{H}), 1.82(\mathrm{~s}, 3 \mathrm{H}), 1.48-1.40(\mathrm{~m}, 2 \mathrm{H}), 1.36-1.27(\mathrm{~m}, 2 \mathrm{H})$, $0.88(\mathrm{t}, J=7.2 \mathrm{~Hz}, 3 \mathrm{H}) ;{ }^{1} \mathrm{H} \operatorname{NMR}\left(400 \mathrm{MHz}, \mathrm{CDCl}_{3}\right) \delta: 7.53(\mathrm{~d}$, $J=8.0 \mathrm{~Hz}, 2 \mathrm{H}), 7.43(\mathrm{~d}, J=8.0 \mathrm{~Hz}, 2 \mathrm{H}), 6.35(\mathrm{~d}, J=16.0 \mathrm{~Hz}, 1 \mathrm{H}), 5.63$ $(\mathrm{dd}, J=16.0,8.0 \mathrm{~Hz}, 1 \mathrm{H}), 4.45-4.37(\mathrm{~m}, 1 \mathrm{H}), 3.50-3.46(\mathrm{~m}, 1 \mathrm{H}), 3.42$ (s, 3H), 3.37-3.30 (m, 1H), $2.5(\mathrm{bs}, 1 \mathrm{H}), 2.44(\mathrm{t}, J=8.0 \mathrm{~Hz}, 2 \mathrm{H}), 1.92$ $(\mathrm{s}, 3 \mathrm{H}), 1.52-1.35(\mathrm{~m}, 4 \mathrm{H}), 0.92(\mathrm{t}, J=8.0 \mathrm{~Hz}, 3 \mathrm{H}) ;{ }^{13} \mathrm{C} \mathrm{NMR}$ $\left(101 \mathrm{MHz}, \mathrm{CDCl}_{3}\right) \delta: 208.8,140.9,130.3,128.5\left(\mathrm{q}, J_{C-F}=32 \mathrm{~Hz}\right), 127.1$, 126.3, 125.2 (q, $\left.J_{C-F}=16 \mathrm{~Hz}\right), 124.3$ (q, $\left.J_{C-F}=270 \mathrm{~Hz}\right), 104.7,103.1$, 76.5, 71.2, 59.1, 29.9, 29.8, 22.4, 15.2, 14.0; ${ }^{19} \mathrm{~F}$ NMR (326.27 MHz, $\left.\mathrm{CDCl}_{3}\right) \delta$ : -62.4 ; FTIR $\left(v_{\max } / \mathrm{cm}^{-1}\right): 3435,2941,2862,1609,1326$, 1160, 1133, 1071, 833, 612; MS (EI, $m / z): 354\left(<1, \mathrm{M}^{+}\right), 309(35)$, 291(30), 267(45), 249(60), 237(90), 159(85), 109(45), 55(40), 45(100); HRMS (ESI) $\mathrm{C}_{20} \mathrm{H}_{25} \mathrm{~F}_{3} \mathrm{O}_{2}[\mathrm{M}+\mathrm{H}]^{+}: 355.1784$ (calculated); 355.1781 (found).

\subsubsection{2. (2S,6S,E)-7-(3-chlorophenyl)-1-methoxy-5-methylundeca- 3,5,6-trien-2-ol (3bh)}

Isolated as a 90:10 mixture of diastereomers; ${ }^{1} \mathrm{H}$ NMR $(400 \mathrm{MHz}$, $\left.\mathrm{C}_{6} \mathrm{D}_{6}\right) \delta: 7.53(\mathrm{t}, J=2.0 \mathrm{~Hz}, 1 \mathrm{H}), 7.17(\mathrm{dd}, J=1.6,0.8 \mathrm{~Hz}, 1 \mathrm{H}), 7.04$ (ddd, $J=7.9,2.1 .1 .0 \mathrm{~Hz}, 1 \mathrm{H}), 6.87(\mathrm{t}, J=8.0 \mathrm{~Hz}, 1 \mathrm{H}), 6.49$ (dd, $J=15.7$, $1.6 \mathrm{~Hz}, 1 \mathrm{H}), 5.60(\mathrm{dd}, J=15.7,5.9 \mathrm{~Hz}, 1 \mathrm{H}), 4.31-4.29(\mathrm{~m}, 1 \mathrm{H}), 3.12$ (dd, A of ABX, $J_{A B}=9.4 \mathrm{~Hz}, J_{A X}=7.7 \mathrm{~Hz}, 1 \mathrm{H}$ ), 3.08 (dd, B of ABX, $\left.J_{A B}=9.4 \mathrm{~Hz}, J_{B X}=3.7 \mathrm{~Hz}, 1 \mathrm{H}\right), 3.00(\mathrm{~s}, 3 \mathrm{H}), 2.30(\mathrm{bs}, 1 \mathrm{H}), 2.22(\mathrm{t}$, $J=7.6 \mathrm{~Hz}, 2 \mathrm{H}), 1.78(\mathrm{~s}, 3 \mathrm{H}), 1.47-1.38(\mathrm{~m}, 2 \mathrm{H}), 1.27$ (sext, $J=7.6 \mathrm{~Hz}$, $2 \mathrm{H}), 0.82(\mathrm{t}, J=7.2 \mathrm{~Hz}, 3 \mathrm{H}) ;{ }^{13} \mathrm{C}$ NMR $\left(101 \mathrm{MHz}, \mathrm{C}_{6} \mathrm{D}_{6}\right) \delta: 208.8$, 140.2, 135.3, 130.3, 129.7, 129.1, 127.3, 126.96, 126.93,125.24, 105.4, 104.0, 77.4, 71.5, 59.0, 30.6, 30.4, 23.0, 15.7, 14.5; FTIR $\left(v_{\max } / \mathrm{cm}^{-1}\right)$ : 3406, 2930, 1617, 1480, 1109, 972, 783, 686; MS (EI, $m / z): 320(5$, $\mathrm{M}^{+}$), 257(15), 203(45), 165(35), 125(40), 44(100); HRMS (ESI) $\mathrm{C}_{19} \mathrm{H}_{25} \mathrm{ClO}_{2} \mathrm{Na}[\mathrm{M}+\mathrm{Na}]^{+}: 343.1441$ (calculated), 343.1439 (found).

\subsubsection{3. (2S,6S,E)-7-(2-fluorophenyl)-1-methoxy-5-methylundeca- \\ 3,5,6-trien-2-ol (3bi)}

Isolated as a 92:8 mixture of diastereomers; ${ }^{1} \mathrm{H}$ NMR $(400 \mathrm{MHz}$, $\left.\mathrm{C}_{6} \mathrm{D}_{6}\right) \delta: 7.25-7.21(\mathrm{~m}, 1 \mathrm{H}), 6.85-6.80(\mathrm{~m}, 3 \mathrm{H}), 6.59(\mathrm{dd}, J=15.9$, $1.4 \mathrm{~Hz}, 1 \mathrm{H}), 5.59(\mathrm{dd}, J=15.9,5.9 \mathrm{~Hz}, 1 \mathrm{H}), 4.33-4.29(\mathrm{~m}, 1 \mathrm{H}), 3.13$ (dd, A of ABX, $J_{A B}=9.4 \mathrm{~Hz}, J_{A X}=8.2 \mathrm{~Hz}, 1 \mathrm{H}$ ), 3.09 (dd, B of ABX, $\left.J_{A B}=9.4 \mathrm{~Hz}, J_{B X}=3.6 \mathrm{~Hz}, 1 \mathrm{H}\right), 3.00(\mathrm{~s}, 3 \mathrm{H}), 2.47(\mathrm{t}, J=7.4 \mathrm{~Hz}, 2 \mathrm{H})$, 2.30 (bs, $1 \mathrm{H}), 1.85(\mathrm{~s}, 3 \mathrm{H}), 1.52-1.44(\mathrm{~m}, 2 \mathrm{H}), 1.32$ (sext, $J=8.0 \mathrm{~Hz}$, $2 \mathrm{H}), 0.83(\mathrm{t}, J=7.2 \mathrm{~Hz}, 3 \mathrm{H}) ;{ }^{1} \mathrm{H}$ NMR $\left(400 \mathrm{MHz}, \mathrm{CDCl}_{3}\right) \delta: 7.27-7.15$ $(\mathrm{m}, 2 \mathrm{H}), 7.09-6.98(\mathrm{~m}, 2 \mathrm{H}), 6.36(\mathrm{~d}, J=16.0 \mathrm{~Hz}, 1 \mathrm{H}), 5.55(\mathrm{dd}$, $J=16.0,8.0 \mathrm{~Hz}, 1 \mathrm{H}), 4.42-4.37(\mathrm{~m}, 1 \mathrm{H}), 3.49-3.45(\mathrm{~m}, 1 \mathrm{H}), 3.41(\mathrm{~s}$, $3 \mathrm{H}), 3.36-3.31(\mathrm{~m}, 1 \mathrm{H}), 2.42(\mathrm{t}, J=8.0 \mathrm{~Hz}, 2 \mathrm{H}), 1.87(\mathrm{~s}, 3 \mathrm{H}), 1.65(\mathrm{bs}$, 1H), $1.43-1.19(\mathrm{~m}, 4 \mathrm{H}), 0.89(\mathrm{t}, J=8.0 \mathrm{~Hz}, 3 \mathrm{H}) ;{ }^{13} \mathrm{C}$ NMR $(101 \mathrm{MHz}$, $\left.\mathrm{CDCl}_{3}\right) \delta:$ 208.7, $160.2\left(\mathrm{~d}, J_{C-F}=247.3 \mathrm{~Hz}\right), 131.3,129.8$ (d, JC$F=3.8 \mathrm{~Hz}), 128.4\left(\mathrm{~d}, J_{C-F}=8.3 \mathrm{~Hz}\right), 126.4,125.7\left(\mathrm{~d}, J_{C-F}=12.9 \mathrm{~Hz}\right)$, $124.0\left(\mathrm{~d}, J_{C-F}=3.8 \mathrm{~Hz}\right), 116.1\left(\mathrm{~d}, J_{C-F}=22.8 \mathrm{~Hz}\right), 100.7,100.5,76.7$, 71.4, 59.2, 32.0, 30.2, 22.4, 15.5, 14.1; ${ }^{19} \mathrm{~F}$ NMR (326.27 $\left.\mathrm{MHz}, \mathrm{CDCl}_{3}\right)$ $\delta$ : $-114.0 ;$ FTIR $\left(v_{\max } / \mathrm{cm}^{-1}\right): 3425,2923,1487,1440,1196,1110,971$, 752; MS (EI, $m / z): 304\left(5, \mathrm{M}^{+}\right), 259(15), 199(25), 187(60), 133(25)$, 109(100), 45(35); HRMS (ESI) $\mathrm{C}_{19} \mathrm{H}_{26} \mathrm{FO}_{2} \quad[\mathrm{M}+\mathrm{H}]^{+}: 305.1911$ (calculated), 305.1913 (found). 
4.3.14. (2S,6S,E)-1-methoxy-5-methyl-7-(naphthalen-1-yl)undeca3,5,6-trien-2-ol (3bj)

Isolated as a 91:9 mixture of diastereomers; ${ }^{1} \mathrm{H}$ NMR $(400 \mathrm{MHz}$, $\left.\mathrm{C}_{6} \mathrm{D}_{6}\right) \delta: 8.39(\mathrm{~d}, J=8.6 \mathrm{~Hz}, 1 \mathrm{H}), 7.66(\mathrm{dd}, J=8.2,0.8 \mathrm{~Hz}, 1 \mathrm{H}), 7.57(\mathrm{~d}$, $J=8.2 \mathrm{~Hz}, 1 \mathrm{H}), 7.41-7.37(\mathrm{~m}, 2 \mathrm{H}), 7.29-7.24(\mathrm{~m}, 2 \mathrm{H}), 6.67(\mathrm{dd}$, $J=15.8,1.4 \mathrm{~Hz}, 1 \mathrm{H}), 5.54(\mathrm{dd}, J=15.8,5.7 \mathrm{~Hz}, 1 \mathrm{H}), 4.35-4.30(\mathrm{~m}$, $1 \mathrm{H}$ ), 3.13 (dd, A of ABX, $J_{A B}=9.4 \mathrm{~Hz}, J_{A X}=8.1 \mathrm{~Hz}, 1 \mathrm{H}$ ), 3.08 (dd, B of $\left.\mathrm{ABX}, J_{A B}=9.4 \mathrm{~Hz}, J_{B X}=3.7 \mathrm{~Hz}, 1 \mathrm{H}\right), 3.00(\mathrm{~s}, 3 \mathrm{H}), 2.47(\mathrm{td}, J=7.5$, $1.8 \mathrm{~Hz}, 2 \mathrm{H}$ ), $1.84(\mathrm{~s}, 3 \mathrm{H}$ ), 1.524 (quint, $J=7.2 \mathrm{~Hz}, 2 \mathrm{H}$ ), 1.33 (sext, $J=7.6 \mathrm{~Hz}, 2 \mathrm{H}), 0.83(\mathrm{t}, J=7.6 \mathrm{~Hz}, 3 \mathrm{H}) ;{ }^{13} \mathrm{C} \mathrm{NMR}\left(101 \mathrm{MHz}, \mathrm{C}_{6} \mathrm{D}_{6}\right) \delta$ : 207.3, 137.6, 135.0, 132.4, 130.8, 129.2, 128.1, 126.6, 126.4, 126.32, 126.1, 104.5, 100.7, 77.5, 71.6, 58.9, 35.5, 31.0, 23.1, 16.1, 14.5; FTIR $\left(v_{\max } / \mathrm{cm}^{-1}\right): 3435,2922,1946,1470,1206,1129,986,804,782 ;$ MS $(\mathrm{EI}, \mathrm{m} / \mathrm{z}): 336\left(20, \mathrm{M}^{+}\right), 273(30), 261(55), 229(100), 217(65)$, 202(80), 165(60), 141(35), 44(95); HRMS (ESI) $\mathrm{C}_{23} \mathrm{H}_{28} \mathrm{O}_{2} \mathrm{Na}$ $[\mathrm{M}+\mathrm{Na}]^{+}: 359.1982$ (calculated), 359.1983 (found).

\subsubsection{5. (2S,6S,E)-1-methoxy-5-methyl-7-(naphthalen-2-yl)undeca-} 3,5,6-trien-2-ol (3bk)

Isolated as a 91:9 mixture of diastereomers; ${ }^{1} \mathrm{H}$ NMR $(400 \mathrm{MHz}$, $\left.\mathrm{C}_{6} \mathrm{D}_{6}\right): \delta 7.84(\mathrm{~s}, 1 \mathrm{H}), 7.71(\mathrm{dd}, J=8.4,1.6 \mathrm{~Hz}, 1 \mathrm{H}), 7.68-7.60(\mathrm{~m}, 3 \mathrm{H})$, 7.26 (quint, $J=7.1,1.6 \mathrm{~Hz}, 2 \mathrm{H}$ ), 6.62 (dd, $J=15.8,1.4 \mathrm{~Hz}, 1 \mathrm{H}$ ), 5.67 (dd, $J=15.8,5.9 \mathrm{~Hz}, 1 \mathrm{H}), 4.37-4.33(\mathrm{~m}, 1 \mathrm{H}), 3.15(\mathrm{dd}, \mathrm{A}$ of $\mathrm{ABX}$, $\left.J_{A B}=9.4 \mathrm{~Hz}, J_{A X}=7.8 \mathrm{~Hz}, 1 \mathrm{H}\right), 3.12$ (dd, B of ABX, $J_{A B}=9.4 \mathrm{~Hz}$, $\left.J_{B X}=3.5 \mathrm{~Hz}, 1 \mathrm{H}\right), 3.01(\mathrm{~s}, 3 \mathrm{H}), 2.52(\mathrm{t}, J=7.6 \mathrm{~Hz}, 2 \mathrm{H}), 2.35(\mathrm{bs}, 1 \mathrm{H})$, 1.91 (s, 3H), 1.63-1.56 (m, 2H), 1.38 (sext, $J=7.6 \mathrm{~Hz}, 2 \mathrm{H}), 0.89$ (t, $J=7.2 \mathrm{~Hz}, 3 \mathrm{H}) ;{ }^{13} \mathrm{C}$ NMR $\left(101 \mathrm{MHz}, \mathrm{C}_{6} \mathrm{D}_{6}\right): \delta 209.6,135.4,134.7$, 133.5, 130.3, 128.8, 128.7, 126.7, 126.5, 126.3, 124.6, 106.6, 103.8, 77.5, 71.7, 59.0, 30.8, 30.7, 23.2, 16.0, 14.6; FTIR $\left(v_{\max } / \mathrm{cm}^{-1}\right): 3451,2950$, 1449, 1363, 1122, 959, 863, 825, 747; MS (EI, $m / z): 336\left(15, \mathrm{M}^{+}\right)$, 291(30), 261(30), 219(80), 207(55), 165(45), 141(100), 73(50), 45(60); HRMS (ESI) $\mathrm{C}_{23} \mathrm{H}_{28} \mathrm{O}_{2} \mathrm{Na}[\mathrm{M}+\mathrm{Na}]^{+}: 359.1982$ (calculated), 359.1983 (found).

\subsubsection{6. (2S,3E,6S,8E)-7-butyl-1-methoxy-5-methyldodeca-3,5,6,8- tetraen-2-ol (3bl)}

Isolated as a 83:17 mixture of diastereomers; ${ }^{1} \mathrm{H}$ NMR $(400 \mathrm{MHz}$, $\left.\mathrm{C}_{6} \mathrm{D}_{6}\right) \delta: 6.53$ (dd, $\left.J=15.1,1.0 \mathrm{~Hz}, 1 \mathrm{H}\right), 6.09$ (d, $\left.J=16.0 \mathrm{~Hz}, 1 \mathrm{H}\right), 5.65$ $(\mathrm{dt}, J=15.7,7.0 \mathrm{~Hz}, 1 \mathrm{H}), 5.58(\mathrm{dd}, J=15.7,5.6 \mathrm{~Hz}, 1 \mathrm{H}), 4.33-4.29(\mathrm{~m}$, $1 \mathrm{H}$ ), 3.13 (dd, A of ABX, $J_{A B}=9.5 \mathrm{~Hz}, J_{A X}=7.8 \mathrm{~Hz}, 1 \mathrm{H}$ ), 3.09 (dd, B of $\left.\mathrm{ABX}, J_{A B}=9.5 \mathrm{~Hz}, J_{B X}=4.0 \mathrm{~Hz}, 1 \mathrm{H}\right), 3.00(\mathrm{~s}, 3 \mathrm{H}), 2.20(\mathrm{t}, J=7.2 \mathrm{~Hz}$, $2 \mathrm{H}), 2.02(\mathrm{q}, J=6.5 \mathrm{~Hz}, 2 \mathrm{H}), 1.84(\mathrm{~s}, 3 \mathrm{H}), 1.56-1.48(\mathrm{~m}, 2 \mathrm{H})$, $1.39-1.29(\mathrm{~m}, 4 \mathrm{H}), 0.87(\mathrm{t}, J=7.6 \mathrm{~Hz}, 3 \mathrm{H}), 0.85(\mathrm{t}, J=7.6 \mathrm{~Hz}, 3 \mathrm{H}) ;{ }^{13} \mathrm{C}$ NMR $\left(101 \mathrm{MHz}, \mathrm{C}_{6} \mathrm{D}_{6}\right) \delta: 210.5,131.0,129.6,128.7,127.8,105.3,101.2$, 77.5, 71.6, 58.9, 35.8, 30.7, 29.6, 23.4, 23.3, 16.2, 14.6, 14.3; FTIR $\left(v_{\max } / \mathrm{cm}^{-1}\right): 3442,2927,1480,1261,1113,958,796$; MS (EI, $\left.m / z\right)$ : 278(10), 233(25), 161(30), 119(45), 105(100), 93(60), 55(55); HRMS (ESI) $\mathrm{C}_{19} \mathrm{H}_{32} \mathrm{O}_{2} \mathrm{Na} \quad[\mathrm{M}+\mathrm{Na}]^{+}: 315.2319$ (calculated), 315.2320 (found).

\subsubsection{7. (2S,6S,E)-1-methoxy-5-methyl-7-((E)-styryl)undeca-3,5,6- trien-2-ol (3bm)}

Isolated as a 70:30 mixture of diastereomers; ${ }^{1} \mathrm{H}$ NMR $\left(400 \mathrm{MHz}, \mathrm{C}_{6} \mathrm{D}_{6}\right) \delta: 7.27-7.23(\mathrm{~m}, 2 \mathrm{H}), 7.12(\mathrm{t}, J=7.8 \mathrm{~Hz}, 2 \mathrm{H})$, $7.05-7.02(\mathrm{~m}, 1 \mathrm{H}), 6.83(\mathrm{~d}, J=16.4 \mathrm{~Hz}, 1 \mathrm{H}), 6.58(\mathrm{~d}, J=16.4 \mathrm{~Hz}, 1 \mathrm{H})$, 6.57 (dd, $J=15.8,0.4 \mathrm{~Hz}, 1 \mathrm{H}), 5.64$ (ddd, $J=16.0,5.6,0.8 \mathrm{~Hz}, 1 \mathrm{H})$, $5.64(\mathrm{ddd}, J=16.0,5.6,0.8 \mathrm{~Hz}, 1 \mathrm{H}), 4.36-4.31(\mathrm{~m}, 1 \mathrm{H}), 3.14(\mathrm{dd}$, A of $\left.\mathrm{ABX}, J_{A B}=9.4 \mathrm{~Hz}, J_{A X}=7.7 \mathrm{~Hz}, 1 \mathrm{H}\right), 3.10\left(\mathrm{dd}, \mathrm{B}\right.$ of $\mathrm{ABX}, J_{A B}=9.4 \mathrm{~Hz}$, $\left.J_{B X}=3.6 \mathrm{~Hz}, 1 \mathrm{H}\right), 3.00(\mathrm{~s}, 3 \mathrm{H}), 2.26(\mathrm{t}, J=7.2 \mathrm{~Hz}, 2 \mathrm{H}), 1.86(\mathrm{~s}, 3 \mathrm{H})$, $1.57-1.50(\mathrm{~m}, 2 \mathrm{H}), 1.39-1.30(\mathrm{~m}, 2 \mathrm{H}), 0.88(\mathrm{t}, J=7.6 \mathrm{~Hz}, 3 \mathrm{H}) ;{ }^{13} \mathrm{C}$ NMR $\left(101 \mathrm{MHz}, \mathrm{C}_{6} \mathrm{D}_{6}\right) \delta: 212.4,138.4,130.4,129.2,127.84,127.80$, 127.0, 106.0, 101.6, 77.4, 71.6, 59.0, 30.6, 29.4, 23.2, 16.1, 14.6; FTIR $\left(v_{\max } / \mathrm{cm}^{-1}\right): 3435,2923,1460,1142,957,753,693$; MS (EI, $\left.m / z\right)$ : 312(40), 267(35), 223(15), 195(80), 165(60), 115(50), 91(100), 32(60); HRMS (ESI) $\mathrm{C}_{21} \mathrm{H}_{29} \mathrm{O}_{2}[\mathrm{M}+\mathrm{H}]^{+}: 313.2161$ (calculated),

\subsection{8 (found)}

4.3.18. (E)-4-Methyl-6-phenyldeca-2,4,5-trien-1-ol (3ca)

${ }^{1} \mathrm{H}$ NMR $\left(400 \mathrm{MHz}, \mathrm{C}_{6} \mathrm{D}_{6}\right) \delta: 7.45(\mathrm{~d}, J=8.0 \mathrm{~Hz}, 2 \mathrm{H}), 7.19(\mathrm{t}$, $J=7.8 \mathrm{~Hz}, 2 \mathrm{H}), 7.07(\mathrm{td}, J=7.6,0.8 \mathrm{~Hz}, 1 \mathrm{H}), 6.29(\mathrm{~d}, J=15.6 \mathrm{~Hz}, 1 \mathrm{H})$, $5.59(\mathrm{dtd}, J=15.6,5.8,0.8 \mathrm{~Hz}, 1 \mathrm{H}), 3.90(\mathrm{~d}, J=5.8 \mathrm{~Hz}, 2 \mathrm{H}), 2.41(\mathrm{t}$, $7.4 \mathrm{~Hz}, 2 \mathrm{H}), 1.83(\mathrm{~s}, 3 \mathrm{H}), 1.54$ (quin, $J=7.4 \mathrm{~Hz}, 2 \mathrm{H}), 1.39-1.30(\mathrm{~m}, 2 \mathrm{H})$, $0.87(\mathrm{t}, J=7.6 \mathrm{~Hz}, 3 \mathrm{H}) ;{ }^{13} \mathrm{C}$ NMR $\left(101 \mathrm{MHz}, \mathrm{C}_{6} \mathrm{D}_{6}\right) \delta: 208.6,138.0$, 129.7, 129.12, 129.07, 127.4, 127.0, 106.3, 103.4, 63.8, 30.8, 30.7, 23.2, 15.9, 14.5; FTIR $\left(v_{\max } / \mathrm{cm}^{-1}\right): 3345,2912,1488,1449,1305,978,689$; MS (EI, $m / z): 242\left(<5, \mathrm{M}^{+}\right), 224(5), 200(15), 169(100), 154(20)$, 141(25), 128(20), 115(20), 91(30), 41(5); HRMS (ESI) $\mathrm{C}_{17} \mathrm{H}_{22} \mathrm{ONa}$ $[\mathrm{M}+\mathrm{Na}]^{+}: 265.1563$ (calculated), 265.1569 (found).

\subsubsection{9. (2S,6S,E)-5-methyl-7-phenylundeca-3,5,6-triene-1,2-diol (3da)}

Isolated as a 91:9 mixture of diastereomers; ${ }^{1} \mathrm{H}$ NMR $(400 \mathrm{MHz}$, $\left.\mathrm{C}_{6} \mathrm{D}_{6}\right) \delta$ : 7.45-7.42 (m, 2H), 7.21-7.16 (m, 2 H), 7.08-7.04 (m, 1H), 6.42 (dd, $J=15.6,1.6 \mathrm{~Hz}, 1 \mathrm{H}), 5.50$ (dd, $J=15.6,6.4 \mathrm{~Hz}, 1 \mathrm{H}$ ), 4.05-4.02 (m, 1H), 3.40-3.37 (m, 1H), 3.30-3.24 (m, 1H), $2.40(\mathrm{t}$, $J=7.4 \mathrm{~Hz}, 2 \mathrm{H}), 1.82(\mathrm{~d}, J=0.8 \mathrm{~Hz}, 3 \mathrm{H}), 1.55-1.48(\mathrm{~m}, 2 \mathrm{H}), 1.38-1.28$ $(\mathrm{m}, 2 \mathrm{H}), 0.86(\mathrm{t}, J=7.6 \mathrm{~Hz}, 3 \mathrm{H}) ;{ }^{13} \mathrm{C}$ NMR $\left(101 \mathrm{MHz}, \mathrm{C}_{6} \mathrm{D}_{6}\right) \delta: 208.9$, 137.9, 130.9, 129.1, 127.4, 127.1, 106.4, 103.3, 73.8, 67.2, 30.8, 30.7, 23.2, 15.9, 14.5; MS (EI, $m / z):$ 256(25), 239(27), 112(95), 83(40), 70(75), 57(100), 43(65); FTIR $\left(v_{\max } / \mathrm{cm}^{-1}\right)$ : 3365, 2951, 2920, 2862, $1925,1597,1454,1079,1031,967,750,698$; HRMS (ESI) $\mathrm{C}_{18} \mathrm{H}_{25} \mathrm{O}_{2}$ $[\mathrm{M}+\mathrm{H}]^{+}: 273.1849$ (calculated), 273.1851 (found).

\subsubsection{0. (2S,6S,E)-1-(benzyloxy)-5-methyl-7-phenylundeca-3,5,6- \\ trien-2-ol (3ea)}

Isolated as a 90:10 mixture of diastereomers; ${ }^{1} \mathrm{H}$ NMR $(400 \mathrm{MHz}$, $\left.\mathrm{C}_{6} \mathrm{D}_{6}\right) \delta: 7.44(\mathrm{~d}, J=7.8 \mathrm{~Hz}, 2 \mathrm{H}), 7.20-7.14(\mathrm{~m}, 6 \mathrm{H}), 7.11-7.05(\mathrm{~m}$, $2 \mathrm{H}), 6.57(\mathrm{~d}, J=15.9 \mathrm{~Hz}, 1 \mathrm{H}), 5.60(\mathrm{dd}, J=15.9,5.9 \mathrm{~Hz}, 1 \mathrm{H})$, 4.39-4.34 (m, 1H), $4.24(\mathrm{~s}, 2 \mathrm{H}), 3.28$ (dd, A of ABX, $J_{A B}=9.3 \mathrm{~Hz}$, $\left.J_{A X}=7.9 \mathrm{~Hz}, 1 \mathrm{H}\right), 3.21\left(\mathrm{dd}, \mathrm{B}\right.$ of $\left.\mathrm{ABX}, J_{A B}=9.3 \mathrm{~Hz}, J_{B X}=3.8 \mathrm{~Hz}, 1 \mathrm{H}\right)$, $2.39(\mathrm{t}, J=7.2 \mathrm{~Hz}, 2 \mathrm{H}), 2.25(\mathrm{bs}, 1 \mathrm{H}), 1.85$ (s, 3H), 1.51 (quint, $J=7.4 \mathrm{~Hz}, 2 \mathrm{H}), 1.32$ (sext, $J=7.6 \mathrm{~Hz}, 2 \mathrm{H}), 0.85$ (t, $J=7.2 \mathrm{~Hz}, 3 \mathrm{H}) ;{ }^{13} \mathrm{C}$ NMR (101 MHz, $\left.\mathrm{C}_{6} \mathrm{D}_{6}\right) \delta: 208.8,139.0,137.9,130.5,129.1,129.0$, 128.5, 128.3, 128.2, 127.4, 127.1, 106.3, 103.4, 75.1, 73.6, 71.8, 30.8, 30.7, 23.2, 15.9, 14.5; FTIR $\left(v_{\max } / \mathrm{cm}^{-1}\right): 3435,2915,1503,1450,1142$, 983, 763, 674; MS (EI, $m / z)$ : 362( $<5, \mathrm{M}^{+}$), 320(5), 253(10), 197(20), 181(25), 169(45), 129(20), 91(100), 69(35), 41(40); HRMS (ESI) $\mathrm{C}_{25} \mathrm{H}_{30} \mathrm{O}_{2} \mathrm{Na}[\mathrm{M}+\mathrm{Na}]^{+}: 385.2138$ (calculated), 385.2138 (found).

\subsubsection{1. (2S,6S,E)-1-((tert-butyldimethylsilyl)oxy)-5-methyl-7-}

phenylundeca-3,5,6-trien-2-ol (3fa)

Isolated as a 92:8 mixture of diastereomers; ${ }^{1} \mathrm{H} \mathrm{NMR}(400 \mathrm{MHz}$, $\left.\mathrm{C}_{6} \mathrm{D}_{6}\right) \delta: 7.44(\mathrm{~d}, J=7.6 \mathrm{~Hz}, 2 \mathrm{H}), 7.19(\mathrm{t}, J=8.0 \mathrm{~Hz}, 2 \mathrm{H}), 7.07(\mathrm{t}$, $J=7.4 \mathrm{~Hz}, 1 \mathrm{H}), 6.58(\mathrm{dd}, J=15.9,1.2 \mathrm{~Hz}, 1 \mathrm{H}), 5.65$ (dd, $J=15.9$, $6.1 \mathrm{~Hz}, 1 \mathrm{H}), 4.25$ (bs, $1 \mathrm{H}), 3.53$ (dd, A of $A B X, J_{A B}=9.9 \mathrm{~Hz}$, $\left.J_{A X}=7.3 \mathrm{~Hz}, 1 \mathrm{H}\right), 3.43\left(\mathrm{dd}, \mathrm{B}\right.$ of $\left.A B X, J_{A B}=9.9 \mathrm{~Hz}, J_{B X}=3.7 \mathrm{~Hz}, 1 \mathrm{H}\right)$, $2.39(\mathrm{t}, J=7.6 \mathrm{~Hz}, 2 \mathrm{H}), 2.32(\mathrm{~d}, J=3.1 \mathrm{~Hz}, 1 \mathrm{H}), 1.89(\mathrm{~s}, 3 \mathrm{H}), 1.52$ (quint, $J=7.6 \mathrm{~Hz}, 2 \mathrm{H}), 1.38-1.29(\mathrm{~m}, 2 \mathrm{H}), 0.91(\mathrm{~s}, 9 \mathrm{H}), 0.86(\mathrm{t}$, $J=7.2 \mathrm{~Hz}, 3 \mathrm{H}),-0.01(\mathrm{~s}, 6 \mathrm{H}) ;{ }^{13} \mathrm{C}$ NMR $\left(101 \mathrm{MHz}, \mathrm{C}_{6} \mathrm{D}_{6}\right) \delta: 208.8$, 137.9, 134.9, 131.5, 130.6, 129.1, 127.4, 127.1, 106.3, 103.4, 73.5, 68.2, $30.8,30.7,26.4,23.2,21.9,18.8,16.0,14.5,-4.9$; FTIR $\left(v_{\max } / \mathrm{cm}^{-1}\right)$ : 3426, 2915, 1468, 1318, 1256, 1115, 851, 772, 683; MS (EI, $m / z)$ : 386(<1, $\mathrm{M}^{+}$), 311(5), 237(30), 195(35), 181(55), 169(30), 105(35), 91(55), 75(100); HRMS (ESI) $\mathrm{C}_{24} \mathrm{H}_{39} \mathrm{O}_{2} \mathrm{Si} \quad[\mathrm{M}+\mathrm{H}]^{+}: \quad 387.2714$ (calculated), 387.2716 (found).

\subsubsection{2. (3S,7S,E)-2-methoxy-2,6-dimethyl-8-phenyldodeca-4,6,7- trien-3-ol (3ga)}

Isolated as a 92:8 mixture of diastereomers; ${ }^{1} \mathrm{H}$ NMR $(400 \mathrm{MHz}$, $\left.\mathrm{C}_{6} \mathrm{D}_{6}\right) \delta: 7.44(\mathrm{~d}, J=7.2 \mathrm{~Hz}, 2 \mathrm{H}), 7.43(\mathrm{~d}, J=7.2 \mathrm{~Hz}, 2 \mathrm{H}$, minor $), 7.18(\mathrm{t}$, 
$J=7.6 \mathrm{~Hz}, 2 \mathrm{H}), 7.06(\mathrm{t}, J=7.2 \mathrm{~Hz}, 1 \mathrm{H}), 6.54(\mathrm{~d}, J=15.6 \mathrm{~Hz}, 1 \mathrm{H}), 5.77$ (ddd, $J=15.7,6.6,0.8 \mathrm{~Hz}, 1 \mathrm{H}), 4.06(\mathrm{~d}, J=6.4 \mathrm{~Hz}, 1 \mathrm{H}), 2.92(\mathrm{~s}, 3 \mathrm{H})$, 2.55 (bs, $1 \mathrm{H}$ ), 1.879 (s, 3H), 1.51 (quint, $J=7.6 \mathrm{~Hz}, 2 \mathrm{H}$ ), 1.32 (sext, $J=7.6 \mathrm{~Hz}, 2 \mathrm{H}), 1.03(\mathrm{~s}, 3 \mathrm{H}), 0.97(\mathrm{~s}, 3 \mathrm{H}), 0.85(\mathrm{t}, J=7.6 \mathrm{~Hz}, 3 \mathrm{H}) ;{ }^{13} \mathrm{C}$ NMR (101 MHz, C $\left.{ }_{6} \mathrm{D}_{6}\right) \delta: 208.7,138.0,134.9,131.3,129.1,127.3,127.1$, 106.2, 103.5, 78.7, 78.0, 72.4, 49.4, 30.8, 23.1, 21.9, 21.1, 19.9, 16.0, 14.5; FTIR $\left(v_{\max } / \mathrm{cm}^{-1}\right)$ : 3438, 2920, 1480, 1384, 1067, 962, 761, 708; MS (EI, $m / z)$ : 314( $\left.<1, \mathrm{M}^{+}\right), 242(5), 91(5), 115(5), 73(100)$; HRMS (ESI) $\mathrm{C}_{21} \mathrm{H}_{31} \mathrm{O}_{2}[\mathrm{M}+\mathrm{H}]^{+}: 315.2318$ (calculated), 315.2327 (found).

\subsubsection{3. (2R,6S,E)-5-methyl-7-phenylundeca-3,5,6-trien-2-ol (3ha)}

Isolated as a $91: 9$ mixture of diastereomers; ${ }^{1} \mathrm{H}$ NMR $(400 \mathrm{MHz}$, $\left.\mathrm{C}_{6} \mathrm{D}_{6}\right) \delta: 7.46(\mathrm{~d}, J=8.0 \mathrm{~Hz}, 2 \mathrm{H}), 7.19(\mathrm{t}, J=7.6 \mathrm{~Hz}, 2 \mathrm{H}), 7.07(\mathrm{t}$, $J=7.2 \mathrm{~Hz}, 1 \mathrm{H}), 6.30(\mathrm{~d}, J=15.7 \mathrm{~Hz}, 1 \mathrm{H}), 5.59$ (ddd, $J=15.7,6.4$, $0.8 \mathrm{~Hz}, 1 \mathrm{H}), 4.14-4.08(\mathrm{~m}, 1 \mathrm{H}), 2.42(\mathrm{t}, J=7.2 \mathrm{~Hz}, 2 \mathrm{H}), 1.85(\mathrm{~d}$, $J=0.8 \mathrm{~Hz}, 3 \mathrm{H}$ ), 1.54 (quint, $J=7.2 \mathrm{~Hz}, 2 \mathrm{H}$ ), $1.39-1.30(\mathrm{~m}, 2 \mathrm{H}), 1.12$ (d, $J=6.4 \mathrm{~Hz}, 3 \mathrm{H}), 0.86(\mathrm{t}, J=7.2 \mathrm{~Hz}, 3 \mathrm{H}) ;{ }^{13} \mathrm{C} \operatorname{NMR}\left(101 \mathrm{MHz}, \mathrm{C}_{6} \mathrm{D}_{6}\right) \delta$ : 208.7, 138.0, 134.1, 129.1, 128.3, 127.4, 127.0, 106.2, 103.3, 69.0, 30.8, 30.7, 24.1, 23.15, 16.0, 14.5; FTIR $\left(v_{\max } / \mathrm{cm}^{-1}\right)$ : 3332, 2972, 2920, 1501, 1427, 1089, 982, 782, 708; MS (EI, $m / z): 256\left(<5, \mathrm{M}^{+}\right), 238(5)$, 196(10), 181(15), 169(100), 155(25), 141(25), 129(20), 115(20), 91(25), 77(10), 43(25); HRMS (ESI) $\mathrm{C}_{18} \mathrm{H}_{25} \mathrm{O}[\mathrm{M}+\mathrm{H}]^{+}: 257.1899$ (calculated), 257.1907 (found).

\subsubsection{4. (2S,6S,E)-1-methoxy-5-methyl-7-phenylhepta-3,5,6-trien- 2-ol (3ia)}

Isolated as a $91: 9$ mixture of diastereomers; ${ }^{1} \mathrm{H}$ NMR $(400 \mathrm{MHz}$, $\left.\mathrm{C}_{6} \mathrm{D}_{6}\right) \delta: 7.23(\mathrm{~d}, J=6.8 \mathrm{~Hz}, 2 \mathrm{H}), 7.11(\mathrm{t}, J=7.6 \mathrm{~Hz}, 2 \mathrm{H}), 7.01(\mathrm{t}$, $J=7.2 \mathrm{~Hz}, 1 \mathrm{H}), 6.49$ (d, $J=16.0 \mathrm{~Hz}, 1 \mathrm{H}), 6.20(\mathrm{~s}, 1 \mathrm{H}), 5.62$ (ddt, $J=15.6,5.6,1.2 \mathrm{~Hz}, 1 \mathrm{H}), 4.28$ (bs, $1 \mathrm{H}), 3.11-3.08(\mathrm{~m}, 1 \mathrm{H}), 3.00(\mathrm{~s}$, $3 \mathrm{H}), 3.02-2.90(\mathrm{~m}, 1 \mathrm{H}), 2.16$ (bs, $1 \mathrm{H}), 1.80(\mathrm{~s}, 3 \mathrm{H}) ;{ }^{13} \mathrm{C}$ NMR $\left(101 \mathrm{MHz}, \mathrm{C}_{6} \mathrm{D}_{6}\right) \delta$ : 210.0, 135.4, 129.3, 128.6, 128.3, 127.7, 127.59, 110.7, 104.1, 77.3, 71.6, 59.0, 15.7; FTIR $\left(v_{\max } / \mathrm{cm}^{-1}\right): 3412,2929$, 1506, 1471, 1118, 966, 753, 683; MS (EI, $m / z): 230\left(15, \mathrm{M}^{+}\right), 185(100)$, 165(30), 152(45), 129(60), 115(50), 91(40), 77(30), 45(30); HRMS (ESI) $\mathrm{C}_{15} \mathrm{H}_{19} \mathrm{O}_{2}[\mathrm{M}+\mathrm{H}]^{+}: 231.1379$ (calculated), 231.1385 (found).

\subsubsection{5. (2S,6S,E)-1-methoxy-5-methyl-7-phenylocta-3,5,6-trien-2-} ol (3ja)

Isolated as a $92: 8$ mixture of diastereomers; ${ }^{1} \mathrm{H}$ NMR $(400 \mathrm{MHz}$, $\left.\mathrm{C}_{6} \mathrm{D}_{6}\right) \delta: 7.39(\mathrm{~d}, J=8.4 \mathrm{~Hz}, 2 \mathrm{H}), 7.16(\mathrm{t}, J=7.6 \mathrm{~Hz}, 2 \mathrm{H}), 7.05(\mathrm{t}$, $J=7.6 \mathrm{~Hz}, 1 \mathrm{H}$ ), 6.51 (d, $J=15.6 \mathrm{~Hz}, 1 \mathrm{H}$ ), 5.63 (ddd, $J=15.8,5.9$, $0.8 \mathrm{~Hz}, 1 \mathrm{H}), 4.33-4.29(\mathrm{~m}, 1 \mathrm{H}), 3.13\left(\mathrm{dd}, \mathrm{A}\right.$ of $\mathrm{ABX}, J_{A B}=8.7 \mathrm{~Hz}$, $\left.J_{A X}=6.8 \mathrm{~Hz}, 1 \mathrm{H}\right), 3.11\left(\mathrm{dd}, \mathrm{B}\right.$ of $\left.\mathrm{ABX}, J_{A B}=8.7 \mathrm{~Hz}, J_{B X}=3.4 \mathrm{~Hz}, 1 \mathrm{H}\right)$, 3.01 (s, 3H), 2.36 (bs, $1 \mathrm{H}), 1.97$ (s, 3H), 1.83 (d, $J=0.8 \mathrm{~Hz}, 3 \mathrm{H}) ;{ }^{13} \mathrm{C}$ $\operatorname{NMR}\left(101 \mathrm{MHz}, \mathrm{C}_{6} \mathrm{D}_{6}\right) \delta: 208.9,138.1,130.4,129.0,128.7,127.3,126.7$, 102.2, 101.0, 77.4, 71.7, 59.0, 17.5, 15.8; FTIR $\left(v_{\max } / \mathrm{cm}^{-1}\right): 3432$, 2922, 1498, 1449, 1135, 1027, 978, 767, 689, 593; MS (EI, $m / z)$ : 244(10, $\left.\mathrm{M}^{+}\right), 199(100), 181(35), 166(45), 128(35), 105(20), 91(30)$, 77(20), 45(25); HRMS (ESI) $\mathrm{C}_{16} \mathrm{H}_{21} \mathrm{O}_{2}[\mathrm{M}+\mathrm{H}]^{+}: 245.1535$ (calculated), 245.1536 (found).

4.3.26. (2S,6S,E)-1-methoxy-7-(4-methoxyphenyl)-5-methyl-7phenylhepta-3,5,6-trien-2-ol (3ke)

Isolated as a 91:9 mixture of diastereomers; ${ }^{1} \mathrm{H}$ NMR $(400 \mathrm{MHz}$, $\left.\mathrm{C}_{6} \mathrm{D}_{6}\right) \delta: 7.52-7.46(\mathrm{~m}, 2 \mathrm{H}), 7.40-7.36(\mathrm{~m}, 1 \mathrm{H}), 7.38(\mathrm{~d}, J=8.8 \mathrm{~Hz}$, 1H), 7.18-7.14 (m, 2H), 7.10-7.06 (m, 1H), 6.79-6.75 (m, 1H), 6.77 (d, $J=8.4 \mathrm{~Hz}, 1 \mathrm{H}), 6.58$ (dd, $J=15.8,1.4 \mathrm{~Hz}, 1 \mathrm{H}$ ), 5.63 (dd, $J=15.8$, $5.9 \mathrm{~Hz}, 1 \mathrm{H}), 4.30(\mathrm{q}, J=5.6 \mathrm{~Hz}, 1 \mathrm{H}), 3.29(\mathrm{~s}, 3 \mathrm{H}), 3.11$ (dd, A of ABX, $\left.J_{A B}=8.6 \mathrm{~Hz}, J_{A X}=5.8 \mathrm{~Hz}, 1 \mathrm{H}\right), 3.08\left(\mathrm{dd}, \mathrm{B}\right.$ of $\mathrm{ABX}, J_{A B}=8.6 \mathrm{~Hz}$, $\left.J_{B X}=2.4 \mathrm{~Hz}, 1 \mathrm{H}\right), 3.00(\mathrm{~s}, 3 \mathrm{H}), 2.31$ (bs, $\left.1 \mathrm{H}\right), 1.85(\mathrm{~s}, 3 \mathrm{H}) ;{ }^{13} \mathrm{C} \mathrm{NMR}$ $\left(101 \mathrm{MHz}, \mathrm{C}_{6} \mathrm{D}_{6}\right) \delta: 210.5,160.0,138.2,130.59,130.0,129.9,129.43$, 129.2, 129.1, 127.9, 114.7, 110.3, 103.2, 77.4, 71.6, 59.0, 55.2, 16.0; FTIR $\left(v_{\max } / \mathrm{cm}^{-1}\right): 3432,2931,1507,1267,1180,1122,1036,978,834,767$, 700; MS (EI, $m / z)$ : $336\left(30, \mathrm{M}^{+}\right), 291(100), 262(65), 247(40)$,
183(30), 155(25), 30(25), 45(45); HRMS (ESI) $\mathrm{C}_{22} \mathrm{H}_{25} \mathrm{O}_{3}[\mathrm{M}+\mathrm{H}]^{+}$: 337.1798 (calculated), 337.1799 (found).

4.3.27. Methyl (3R,7S,E)-7-hydroxy-8-methoxy-4-methyl-2phenylocta-2,3,5-trienoate (3la)

Isolated as a 89:11 mixture of diastereomers; ${ }^{1} \mathrm{H}$ NMR $(400 \mathrm{MHz}$, $\left.\mathrm{C}_{6} \mathrm{D}_{6}\right) \delta$ : 7.74-7.70 (m, 2H), 7.18-7.12 (m, 2H), 7.05-7.01 (m, 1H), 6.38 (dd, $J=15.8,1.2 \mathrm{~Hz}, 1 \mathrm{H}), 5.55$ (dd, $J=15.8,5.6 \mathrm{~Hz}, 1 \mathrm{H}$ ) 4.20-4.14 (m, 1H), 3.36 (s, 3H), 3.00-2.93 (m, 2H), $2.94(\mathrm{~s}, 3 \mathrm{H}), 2.04$ (bs, $1 \mathrm{H}), 1.68$ (s, 3H); ${ }^{13} \mathrm{C}$ NMR (101 MHz, $\left.\mathrm{C}_{6} \mathrm{D}_{6}\right) \delta: 216.7,166.5,139.9$, 131.1, 129.4, 129.0, 128.3, 127.0, 105.2, 103.4, 77.1, 71.4, 59.0, 52.2, 15.0; MS (EI, $m / z): 288\left(<5, \mathrm{M}^{+}\right), 256(3), 211(5), 183(5), 155(17), 115$ (8), 89 (4), 77 (9), 51 (5), 45 (100); FTIR $\left(v_{\max } / \mathrm{cm}^{-1}\right)$ : 3419, 2922, 2851, 1926, 1716, 1492, 1434, 1369, 1321, 1273, 1195, 1171, 1123, 1062 , 1039, 964, 918, 898, 781, 694; HRMS (ESI) $\mathrm{C}_{17} \mathrm{H}_{21} \mathrm{O}_{4}[\mathrm{M}+\mathrm{H}]^{+}$: 289.1434 (calculated), 289.1439 (found).

4.3.28. (2S,6S,E)-7-cyclohexyl-1-methoxy-5-methyl-7-

phenylhepta-3,5,6-trien-2-ol (3ma)

Isolated as a 92:8 mixture of diastereomers; ${ }^{1} \mathrm{H}$ NMR $(400 \mathrm{MHz}$, $\left.\mathrm{C}_{6} \mathrm{D}_{6}\right) \delta: 7.43(\mathrm{~d}, J=8.2 \mathrm{~Hz}, 2 \mathrm{H}), 7.19(\mathrm{~d}, J=7.2 \mathrm{~Hz}, 2 \mathrm{H}), 7.08-7.04(\mathrm{~m}$, $1 \mathrm{H}$ ), 6.57 (d, $J=15.7 \mathrm{~Hz}, 1 \mathrm{H}$ ), 5.63 (ddd, $J=15.7,5.9,0.8 \mathrm{~Hz}, 1 \mathrm{H}$ ), $4.36-4.32(\mathrm{~m}, 1 \mathrm{H}), 3.15\left(\mathrm{dd}, \mathrm{A}\right.$ of $\left.A B X, J_{A B}=9.2 \mathrm{~Hz}, J_{A X}=7.7 \mathrm{~Hz}, 1 \mathrm{H}\right)$, $3.11\left(\mathrm{dd}, \mathrm{B}\right.$ of $\left.\mathrm{ABX}, J_{A B}=9.2 \mathrm{~Hz}, J_{B X}=3.6 \mathrm{~Hz}, 1 \mathrm{H}\right), 3.01(\mathrm{~s}, 3 \mathrm{H})$, $1.48-1.41(\mathrm{~m}, 1 \mathrm{H}), 2.34(\mathrm{bs}, 1 \mathrm{H}), 1.96-1.93(\mathrm{~m}, 2 \mathrm{H}), 1.86(\mathrm{~d}$, $J=0.8 \mathrm{~Hz}, 3 \mathrm{H}), 1.72-1.58(\mathrm{~m}, 3 \mathrm{H}), 1.30-1.08(\mathrm{~m}, 5 \mathrm{H}) ;{ }^{13} \mathrm{C} \mathrm{NMR}$ $\left(101 \mathrm{MHz}, \mathrm{C}_{6} \mathrm{D}_{6}\right) \delta: 208.6,137.7,130.7,129.1,128.3,127.6,127.3,112.7$, 104.2, 77.5, 71.7, 59.0, 39.3, 33.8, 33.8, 27.3, 27.3, 27.0, 16.1; FTIR $\left(v_{\max } / \mathrm{cm}^{-1}\right): 3444,2923,2858,1506,1458,1123,953,774,701 ;$ MS (EI, $m / z): 312\left(10, \mathrm{M}^{+}\right), 267(70), 181(100), 141(50), 91(80), 45(60)$; HRMS (ESI) $\mathrm{C}_{21} \mathrm{H}_{29} \mathrm{O}_{2}[\mathrm{M}+\mathrm{H}]^{+}: 313.2161$ (calculated), 313.2178 (found).

\subsubsection{9. (2S,6S,E)-1-methoxy-5,8,8-trimethyl-7-phenylnona-3,5,6- trien-2-ol (3na)}

Isolated as a 92:8 mixture of diastereomers; ${ }^{1} \mathrm{H}$ NMR $(400 \mathrm{MHz}$, $\left.\mathrm{C}_{6} \mathrm{D}_{6}\right) \delta: 7.30-7.27(\mathrm{~m}, 2 \mathrm{H}), 7.14-7.12(\mathrm{~m}, 2 \mathrm{H}), 7.09-7.05(\mathrm{~m}, 1 \mathrm{H})$, 6.59 (dd, $J=15.7,1.2 \mathrm{~Hz}, 1 \mathrm{H}), 5.51$ (dd, $J=16.0,4.3 \mathrm{~Hz}, 1 \mathrm{H})$, $4.35-4.31(\mathrm{~m}, 1 \mathrm{H}), 3.13\left(\mathrm{dd}, \mathrm{A}\right.$ of $\left.\mathrm{ABX}, J_{A B}=9.3 \mathrm{~Hz}, J_{A X}=8.1 \mathrm{~Hz}, 1 \mathrm{H}\right)$, 3.08 (dd, B of $\left.A B X, J_{A B}=9.3 \mathrm{~Hz}, J_{B X}=3.7 \mathrm{~Hz}, 1 \mathrm{H}\right), 3.00(\mathrm{~s}, 3 \mathrm{H}), 2.24$ (bs, 1H), 1.77 (s, 3H), 1.16 (s, 9H); ${ }^{13} \mathrm{C}$ NMR (101 MHz, $\left.\mathrm{C}_{6} \mathrm{D}_{6}\right) \delta: 206.1$, 138.5, 131.2, 130.2, 128.9, 128.5, 127.7, 127.3, 127.1, 100.9, 77.5, 71.6, 58.9, 35.7, 31.8, 30.5, 16.4; FTIR $\left(v_{\max } / \mathrm{cm}^{-1}\right)$ : 3422, 2960, 1460, 1199, 1132, 969, 709; MS (EI, m/z): $286\left(10, \mathrm{M}^{+}\right), 241(75), 197(100)$, 165(35), 141(40), 105(85), 57(95); HRMS (ESI) $\mathrm{C}_{19} \mathrm{H}_{27} \mathrm{O}_{2}[\mathrm{M}+\mathrm{H}]^{+}$: 287.2005 (calculated), 287.2016 (found).

\subsubsection{0. (Z)-1-Methoxy-5,8,8-trimethyl-3-phenylnon-4-en-6-yn-2 ol (4na)}

${ }^{1} \mathrm{H}$ NMR (400 MHz, $\left.\mathrm{C}_{6} \mathrm{D}_{6}\right) \delta: 7.40(\mathrm{~d}, 7.8 \mathrm{~Hz}, 2 \mathrm{H}), 7.20(\mathrm{~d}$ $J=7.6 \mathrm{~Hz}, 2 \mathrm{H}), 7.07(\mathrm{t}, J=7.6 \mathrm{~Hz}, 1 \mathrm{H}), 6.12(\mathrm{dd}, J=9.6,1.4 \mathrm{~Hz}, 1 \mathrm{H})$ $4.26(\mathrm{dd}, J=9.8,6.0 \mathrm{~Hz}, 1 \mathrm{H}), 4.10-4.06(\mathrm{~m}, 1 \mathrm{H}), 3.30$ (dd, A of ABX, $\left.J_{A B}=9.4 \mathrm{~Hz}, J_{A X}=7.1 \mathrm{~Hz}, 1 \mathrm{H}\right), 3.23\left(\mathrm{dd}, \mathrm{B}\right.$ of $\mathrm{ABX}, J_{A B}=9.4 \mathrm{~Hz}$, $\left.J_{B X}=3.4 \mathrm{~Hz}, 1 \mathrm{H}\right), 3.00(\mathrm{~s}, 3 \mathrm{H}), 2.22(\mathrm{~s}, 1 \mathrm{H}), 1.80$ (s, 3H), 1.23 (s, 9H); ${ }^{13} \mathrm{C}$ NMR $\left(101 \mathrm{MHz}, \mathrm{C}_{6} \mathrm{D}_{6}\right) \delta: 143.4,136.4,129.1,128.3,127.0,121.1$, $102.8,79.7,75.8,74.6,59.0,50.4,31.6,28.6,24.0 ;$ FTIR $\left(v_{\max } / \mathrm{cm}^{-1}\right)$ : 3446, 2910, 1459, 1364, 1117, 1079, 688; MS (EI, $m / z)$ : 268(5), 212(100), 197(65), 169(65), 155(95), 141(35), 91(55), 41(40).

\subsubsection{1. (2S,6S,E)-1-methoxy-7-phenylundeca-3,5,6-trien-2-ol (3oa)}

Isolated as a 89:11 mixture of diastereomers; ${ }^{1} \mathrm{H}$ NMR $(400 \mathrm{MHz}$, $\left.\mathrm{C}_{6} \mathrm{D}_{6}\right) \delta: 7.44(\mathrm{~d}, J=7.0 \mathrm{~Hz}, 2 \mathrm{H}), 7.18(\mathrm{t}, J=7.2 \mathrm{~Hz}, 2 \mathrm{H}), 7.06(\mathrm{t}$, $J=7.2 \mathrm{~Hz}, 1 \mathrm{H}), 6.37(\mathrm{ddd}, J=15.3,10.6,1.2 \mathrm{~Hz}, 1 \mathrm{H}), 6.16(\mathrm{dt}, J=10.6$, $2,9 \mathrm{~Hz}, 1 \mathrm{H}$ ), 5.61 (dd, $J=15.5,5.7 \mathrm{~Hz}, 1 \mathrm{H}), 4.27-4.23$ (m, 1H), 3.09 (dd, A of ABX, $J_{A B}=9.4 \mathrm{~Hz}, J_{A X}=8.1 \mathrm{~Hz}, 1 \mathrm{H}$ ), 3.05 (dd, B of ABX, 
$\left.J_{A B}=9.4 \mathrm{~Hz}, J_{B X}=3.6 \mathrm{~Hz}, 1 \mathrm{H}\right), 2.98(\mathrm{~s}, 3 \mathrm{H}), 2.39-2.34(\mathrm{~m}, 2 \mathrm{H}), 2.24$ (bs, $1 \mathrm{H}$ ), 1.52 (quint, $J=7.2 \mathrm{~Hz}, 2 \mathrm{H}$ ), 1.32 (sext, $J=7.6 \mathrm{~Hz}, 2 \mathrm{H}$ ), 0.85 (t, $J=7.6 \mathrm{~Hz}, 3 \mathrm{H}) ;{ }^{13} \mathrm{C}$ NMR $\left(101 \mathrm{MHz}, \mathrm{C}_{6} \mathrm{D}_{6}\right) \delta: 209.0,137.3,131.6,129.1$, 128.5, 127.5, 127.1, 108.2, 97.6, 77.2, 71.3, 58.9, 30.8, 30.5, 23.2, 14.5; FTIR $\left(v_{\max } / \mathrm{cm}^{-1}\right): 3426,2932,1450,1133,974,772,701 ; \mathrm{MS}$ (EI, $\mathrm{m} /$ $z)$ : 272(<5, $\left.\mathrm{M}^{+}\right)$; 254(5), 227(20), 209(20), 185(65), 167(55), 155(75), 141(70), 129(65), 115(70), 91(100), 77(30), 45(50).

\subsubsection{2. (2S,6S,E)-5-butyl-1-((tert-butyldimethylsilyl)oxy)-7-} phenylundeca-3,5,6-trien-2-ol (3pa)

Isolated as a $92: 8$ mixture of diastereomers; ${ }^{1} \mathrm{H}$ NMR $(400 \mathrm{MHz}$, $\left.\mathrm{C}_{6} \mathrm{D}_{6}\right) \delta: 7.48(\mathrm{~d}, J=8.8 \mathrm{~Hz}, 2 \mathrm{H}), 7.20(\mathrm{t}, J=7.6 \mathrm{~Hz}, 2 \mathrm{H}), 7.07(\mathrm{t}$, $J=6.8 \mathrm{~Hz}, 1 \mathrm{H}), 6.54(\mathrm{~d}, J=15.7 \mathrm{~Hz}, 1 \mathrm{H}), 5.80(\mathrm{ddd}, J=15.7,6.0$, $0.4 \mathrm{~Hz}, 1 \mathrm{H}), 4.27$ (bs, $1 \mathrm{H}$ ), 3.55 (dd, A of $\mathrm{ABX}, J_{A B}=9.8 \mathrm{~Hz}$, $\left.J_{A X}=7.6 \mathrm{~Hz}, 1 \mathrm{H}\right), 3.45\left(\mathrm{dd}, \mathrm{B}\right.$ of $\left.\mathrm{ABX}, J_{A B}=9.8 \mathrm{~Hz}, J_{B X}=3.4 \mathrm{~Hz}, 1 \mathrm{H}\right)$, $2.44(\mathrm{t}, J=7.2 \mathrm{~Hz}, 2 \mathrm{H}), 2.29(\mathrm{t}, J=8.0 \mathrm{~Hz}, 2 \mathrm{H}), 1.64-1.54(\mathrm{~m}, 4 \mathrm{H})$, $1.40-1.29(\mathrm{~m}, 4 \mathrm{H}), 0.91(\mathrm{~s}, 9 \mathrm{H}), 0.87(\mathrm{t}, J=7.6 \mathrm{~Hz}, 3 \mathrm{H}), 0.85(\mathrm{t}$, $J=7.6 \mathrm{~Hz}, 3 \mathrm{H}),-0.01(\mathrm{~s}, 6 \mathrm{H}) ;{ }^{13} \mathrm{C}$ NMR $\left(101 \mathrm{MHz}, \mathrm{C}_{6} \mathrm{D}_{6}\right) \delta: 208.2$, $137.9,130.2,129.1,128.3,127.4,126.9,108.6,108.0,73.6,68.2,31.0$, $30.9,30.8,29.8,26.4,23.5,23.3,18.8,14.5,14.5,-4.9$; MS (EI, $m / z)$ : 371(10), 336(5), 315(20), 296(25), 279(25), 212(20), 168(45), 116(60), 91(55), 75(100), 56(95); FTIR $\left(v_{\max } / \mathrm{cm}^{-1}\right)$ : 3437, 2917, 1451, 1272, 1093, 849, 776, 685. HRMS (ESI) $\mathrm{C}_{27} \mathrm{H}_{44} \mathrm{O}_{2} \mathrm{SiNa}^{+}$ $[\mathrm{M}+\mathrm{Na}]^{+}: 451.3003$ (calculated), 451.300 (found).

\subsubsection{3. (2S,6S,E)-5-cyclohexyl-1-methoxy-7-phenylundeca-3,5,6- trien-2-ol (3qa)}

Isolated as a 88:12 mixture of diastereomers; ${ }^{1} \mathrm{H}$ NMR $\left(400 \mathrm{MHz}, \mathrm{C}_{6} \mathrm{D}_{6}\right) \delta: 7.51(\mathrm{~d}, J=7.4 \mathrm{~Hz}, 2 \mathrm{H}), 7.20(\mathrm{t}, J=7.6 \mathrm{~Hz}, 2 \mathrm{H})$, $7.07(\mathrm{t}, J=7.2 \mathrm{~Hz}, 1 \mathrm{H}), 6.39$ (dd, $J=16.0,1.2 \mathrm{~Hz}, 1 \mathrm{H}), 5.86$ (dd, $J=16.0,5.9 \mathrm{~Hz}, 1 \mathrm{H}), 4.35-4.31(\mathrm{~m}, 1 \mathrm{H}), 3.14(\mathrm{dd}, \mathrm{A}$ of $\mathrm{ABX}$, $\left.J_{A B}=9.5 \mathrm{~Hz}, J_{A X}=8.9 \mathrm{~Hz}, 1 \mathrm{H}\right), 3.10\left(\mathrm{dd}, \mathrm{B}\right.$ of $\mathrm{ABX}, J_{A B}=9.5 \mathrm{~Hz}$, $\left.J_{B X}=4.0 \mathrm{~Hz}, 1 \mathrm{H}\right), 2.99(\mathrm{~s}, 3 \mathrm{H}), 2.46-2.42(\mathrm{~m}, 2 \mathrm{H}), 2.29(\mathrm{bs}, 1 \mathrm{H})$, 2.26-2.22 (m, 1H), 2.07-2.04 (m, 2H), 1.71-1.56 (m, 4H), 1.39-1.06 $(\mathrm{m}, 7 \mathrm{H}), 0.88(\mathrm{t}, J=7.2 \mathrm{~Hz}, 3 \mathrm{H}) ;{ }^{13} \mathrm{C}$ NMR $\left(101 \mathrm{MHz}, \mathrm{C}_{6} \mathrm{D}_{6}\right) \delta: 207.1$, 138.0, 129.1, 128.9, 128.3, 127.3, 126.7, 114.4, 109.0, 77.5, 71.8, 58.9, 39.3, 33.8, 33.7, 31.1, 30.8, 27.3, 27.3, 27.0, 23.4, 14.5; FTIR ( $\left.v_{\max }\right)$ $\left.\mathrm{cm}^{-1}\right)$ : 3446, 2918, 2846, 1424, 1142, 1000, 777, 683; MS (EI, $\left.\mathrm{m} / \mathrm{z}\right)$ : 336(10), 309(35), 279(45), 207(40), 155(50), 91(100), 55(55), 32(55); HRMS (ESI) $\mathrm{C}_{24} \mathrm{H}_{35} \mathrm{O}_{2}[\mathrm{M}+\mathrm{H}]^{+}: 355.2631$ (calculated), 355.2642 (found).

\subsubsection{4. (Z)-5-Cyclohexyl-1-methoxy-3-phenylundec-4-en-6-yn-2- ol (4qa)}

${ }^{1} \mathrm{H}$ NMR $\left(400 \mathrm{MHz}, \mathrm{C}_{6} \mathrm{D}_{6}\right) \delta: 7.45(\mathrm{~d}, J=7.8 \mathrm{~Hz}, 2 \mathrm{H}), 7.20(\mathrm{t}$, $J=7.8 \mathrm{~Hz}, 2 \mathrm{H}), 7.08(\mathrm{t}, J=7.2 \mathrm{~Hz}, 1 \mathrm{H}), 6.22(\mathrm{~d}, J=9.8 \mathrm{~Hz}, 1 \mathrm{H}), 4.34$ $(\mathrm{dd}, J=9.8,5.5 \mathrm{~Hz}, 1 \mathrm{H}), 4.14(\mathrm{bs}, 1 \mathrm{H}), 3.39-3.25(\mathrm{~m}, 2 \mathrm{H}), 3.04(\mathrm{~s}, 3 \mathrm{H})$, $2.21(\mathrm{t}, J=6.4 \mathrm{~Hz}, 2 \mathrm{H}), 2.08(\mathrm{t}, J=12.0 \mathrm{~Hz}, 1 \mathrm{H}), 1.84(\mathrm{dd}, J=26.4$, $12.4 \mathrm{~Hz}, 2 \mathrm{H}), 1.71-1.65(\mathrm{~m}, 2 \mathrm{H}), 1.58-1.29(\mathrm{~m}, 7 \mathrm{H}), 1.22-1.08(\mathrm{~m}$, $3 \mathrm{H}), 0.82(\mathrm{t}, J=6.8 \mathrm{~Hz}, 3 \mathrm{H}) ;{ }^{13} \mathrm{C}$ NMR $\left(101 \mathrm{MHz}, \mathrm{C}_{6} \mathrm{D}_{6}\right) \delta: 143.6$, 132.2, 129.1, 129.0, 128.3, 127.0, 95.9, 79.6, 75.9, 74.6, 59.1, 49.9, 46.4, $33.1,32.9,31.7,27.1,27.0,26.8,22.6,19.9,14.1 ;$ FTIR $\left(v_{\max } / \mathrm{cm}^{-1}\right)$ : 3469, 2940, 1459, 1269, 1089, 876, 697; MS (EI, $m / z): 336(<5)$, 279(65), 223(55), 197(60), 155(100), 115(40), 91(85), 55(35).

\subsubsection{5. (2S,6R,E)-5-(tert-butyl)-1-methoxy-7-phenylundeca-3,5,6-} trien-2-ol (3ra)

Isolated as a $85: 15$ mixture of diastereomers; ${ }^{1} \mathrm{H}$ NMR $(400 \mathrm{MHz}$, $\left.\mathrm{C}_{6} \mathrm{D}_{6}\right) \delta: 7.52(\mathrm{~d}, J=7.6 \mathrm{~Hz}, 2 \mathrm{H}), 7.21(\mathrm{t}, J=7.6 \mathrm{~Hz}, 2 \mathrm{H}), 7.06(\mathrm{t}$, $J=8.0 \mathrm{~Hz}, 1 \mathrm{H}), 6.42(\mathrm{dd}, J=15.5,1.4 \mathrm{~Hz}, 1 \mathrm{H}), 6.02(\mathrm{dd}, J=15.6$, $5.2 \mathrm{~Hz}, 1 \mathrm{H}), 4.31$ (bs, $1 \mathrm{H}$ ), 3.04 (dd, A of $A B X, J_{A B}=7.7 \mathrm{~Hz}$, $\left.J_{A X}=7.3 \mathrm{~Hz}, 1 \mathrm{H}\right), 3.01\left(\mathrm{dd}, \mathrm{B}\right.$ of $\left.A B X, J_{A B}=7.7 \mathrm{~Hz}, J_{B X}=1.3 \mathrm{~Hz}, 1 \mathrm{H}\right)$, $2.93(\mathrm{~s}, 3 \mathrm{H}), 2.47-2.42(\mathrm{~m}, 2 \mathrm{H}), 2.24$ (bs, $1 \mathrm{H}), 1.61$ (quint, $J=7.6 \mathrm{~Hz}$, 2H), 1.55 (s, 3H), 1.39-1.33 (m, 2H), 1.19 (s, 9H), 0.89 (t, $J=7.6 \mathrm{~Hz}$, $3 \mathrm{H}) ;{ }^{3} \mathrm{C}$ NMR $\left(101 \mathrm{MHz}, \mathrm{C}_{6} \mathrm{D}_{6}\right): \delta 203.0,138.2,130.8,129.2,128.3$,
127.2, 126.4, 125.7, 117.3, 109.3, 77.4, 71.5, 58.8, 34.9, 31.0, 30.8, 30.1, 23.4, 14.6; FTIR $\left(v_{\max } / \mathrm{cm}^{-1}\right): 3421,2867,1453,1379,1242,1155,806$, 701; MS (EI, $m / z): 309(<5), 253(20), 212(20), 197(65), 155(95)$, 140(60), 105(40), 91(100), 69(40), 57(95).

\subsubsection{6. (Z)-5-(tert-butyl)-1-methoxy-3-phenylundec-4-en-6-yn-2- ol (4ra)}

Isolated as a $83: 17$ mixture of diastereomers; ${ }^{1} \mathrm{H}$ NMR $(400 \mathrm{MHz}$, $\left.\mathrm{C}_{6} \mathrm{D}_{6}\right) \delta: 7.44(\mathrm{~d}, 7.8 \mathrm{~Hz}, 2 \mathrm{H}), 7.20(\mathrm{~d}, J=8.0 \mathrm{~Hz}, 2 \mathrm{H}), 7.06(\mathrm{td}, J=8.0$, $1.2 \mathrm{~Hz}, 1 \mathrm{H}), 6.33(\mathrm{~d}, J=9.8 \mathrm{~Hz}, 1 \mathrm{H}), 4.37(\mathrm{dd}, J=9.6,5.7 \mathrm{~Hz}, 1 \mathrm{H})$, $4.17-4.12(\mathrm{~m}, 1 \mathrm{H}), 3.34\left(\mathrm{dd}, \mathrm{A}\right.$ of $\left.A B X, J_{A B}=9.3 \mathrm{~Hz}, J_{A X}=7.3 \mathrm{~Hz}, 1 \mathrm{H}\right)$, $3.28\left(\mathrm{dd}, \mathrm{B}\right.$ of $\left.\mathrm{ABX}, J_{A B}=9.3 \mathrm{~Hz}, J_{B X}=3.6 \mathrm{~Hz}, 1 \mathrm{H}\right), 3.02(\mathrm{~s}, 3 \mathrm{H}), 2.28(\mathrm{~s}$, $1 \mathrm{H}), 2.21(\mathrm{t}, J=6.5 \mathrm{~Hz}, 2 \mathrm{H}), 1.44-1.31(\mathrm{~m}, 4 \mathrm{H}), 1.22(\mathrm{~s}, 9 \mathrm{H}), 0.82(\mathrm{t}$, $J=7.6 \mathrm{~Hz}, 3 \mathrm{H}) ;{ }^{13} \mathrm{C}$ NMR $\left(101 \mathrm{MHz}, \mathrm{C}_{6} \mathrm{D}_{6}\right) \delta: 143.6,136.1,131.7,129.1$, 129.0, 127.0, 96.5, 79.5, 75.9, 74.6, 59.0, 50.2, 36.5, 31.7, 29.9, 22.6, $19.8,14.1$; FTIR $\left(v_{\max } / \mathrm{cm}^{-1}\right): 3452,2958,2923,1468,1354,1115$, 763, 710; MS (EI, $m / z)$ : 328(<1, $\left.\mathrm{M}^{+}\right), 310(5), 253(25), 197(65)$, 155(100), 91(40), 57(25).

\subsubsection{1-Methoxy-3-(2-(2-phenylhex-1-en-1-yl)cyclohex-1-en-1- yl)propan-2-one (5sa)}

(E): ${ }^{1} \mathrm{H}$ NMR $\left(400 \mathrm{MHz}, \mathrm{C}_{6} \mathrm{D}_{6}\right) \delta: 7.35(\mathrm{~d}, J=7.6 \mathrm{~Hz}, 2 \mathrm{H}), 7.20-7.16$ $(\mathrm{m}, 2 \mathrm{H}), 7.11(\mathrm{~d}, J=8.0 \mathrm{~Hz}, 1 \mathrm{H}), 6.14(\mathrm{~s}, 1 \mathrm{H}), 3.61(\mathrm{~d}, J=0.8 \mathrm{~Hz}, 2 \mathrm{H})$, $3.15(\mathrm{~s}, 2 \mathrm{H}), 3.02(\mathrm{~s}, 3 \mathrm{H}), 2.51(\mathrm{t}, J=7.2 \mathrm{~Hz}, 2 \mathrm{H}), 2.08(\mathrm{~d}, J=9.2 \mathrm{~Hz}$, $4 \mathrm{H}), 1.58$ (bs, $4 \mathrm{H}), 1.35-1.21(\mathrm{~m}, 4 \mathrm{H}), 0.80(\mathrm{t}, J=6.4 \mathrm{~Hz}, 3 \mathrm{H}) ;{ }^{13} \mathrm{C}$ NMR $\left(101 \mathrm{MHz}, \mathrm{C}_{6} \mathrm{D}_{6}\right) \delta$ : 206.0, 142.9, 142.5, 133.5, 129.0, 128.7, 128.3, 128.1, 127.2, 78.0, 59.2, 45.3, 39.4, 31.1, 30.7, 30.4, 23.6, 23.4, 22.8, 14.4; FTIR $\left(v_{\max } / \mathrm{cm}^{-1}\right): 2922,1738,1440,1209,1094,767,700$; MS (EI, $m / z): 326\left(10, \mathrm{M}^{+}\right)$: 281(25), 238(95), 181(55), 129(30), 91(100), 45(35).

(Z): ${ }^{1} \mathrm{H}$ NMR (400 MHz, $\left.\mathrm{C}_{6} \mathrm{D}_{6}\right) \delta: 7.31(\mathrm{~d}, J=8.0 \mathrm{~Hz}, 2 \mathrm{H}), 7.20-7.16$ (m, 2H), 7.08-7.03 (m, 1H), $6.12(\mathrm{~s}, 1 \mathrm{H}), 3.67(\mathrm{~d}, J=0.8 \mathrm{~Hz}, 2 \mathrm{H}), 3.12$ (s, 2H), 3.08 (s, 3H), 2.40 (t, $J=7.2 \mathrm{~Hz}, 2 \mathrm{H}), 1.96$ (bs, 2H), 1.87 (bs, $2 \mathrm{H}), 1.48-1.36(\mathrm{~m}, 4 \mathrm{H}), 1.38(\mathrm{~s}, 4 \mathrm{H}), 0.82(\mathrm{t}, J=6.4 \mathrm{~Hz}, 3 \mathrm{H}) ;{ }^{13} \mathrm{C}$ NMR $\left(101 \mathrm{MHz}, \mathrm{C}_{6} \mathrm{D}_{6}\right) \delta$ : 205.8, 143.1, 142.0, 133.5, 129.5, 128.5, 128.0, 127.7, 78.0, 59.2, 45.2, 39.4, 31.3, 31.0, 30.4, 23.7, 23.4, 22.8, 14.4; FTIR $\left(v_{\max } / \mathrm{cm}^{-1}\right): 2922,1738,1440,1209,1094,767,700 ; \mathrm{MS}(\mathrm{EI}, \mathrm{m} /$ $z)$ : 326(20, $\left.\mathrm{M}^{+}\right)$: 281(25), 238(95), 181(60), 141(35), 91(100), 45(35).

4.3.38. (3aS,7S,7aS,E)-7-((R)-1-hydroxy-2-methoxyethyl)-5methyl-2-phenyl-4-(1-phenylpentylidene)-3a,4,7,7a-tetrahydro$1 \mathrm{H}$-isoindole-1,3(2H)-dione (6ba)

Isolated as a 91:9 mixture of diastereomers; ${ }^{1} \mathrm{H} \mathrm{NMR}(400 \mathrm{MHz}$, $\left.\mathrm{CDCl}_{3}\right) \delta: 7.46-7.42(\mathrm{~m}, 3 \mathrm{H}), 7.37(\mathrm{t}, J=7.6 \mathrm{~Hz}, 3 \mathrm{H}), 7.32-7.29(\mathrm{~m}$, $2 \mathrm{H}), 7.15(\mathrm{~d}, J=7.6 \mathrm{~Hz}, 2 \mathrm{H}), 6.00(\mathrm{~d}, J=2.4 \mathrm{~Hz}, 1 \mathrm{H}), 4.30(\mathrm{q}$, $J=5.5 \mathrm{~Hz}, 1 \mathrm{H}), 3.93(\mathrm{~d}, J=8.6 \mathrm{~Hz}, 1 \mathrm{H}), 3.64-3.56(\mathrm{~m}, 2 \mathrm{H}), 3.41(\mathrm{~s}$, $3 \mathrm{H}), 3.14(\mathrm{dd}, J=8.6,5.9 \mathrm{~Hz}, 1 \mathrm{H}), 2.56-2.48(\mathrm{~m}, 1 \mathrm{H}), 2.40-2.33(\mathrm{~m}$, $2 \mathrm{H}), 2.06(\mathrm{t}, J=1.6 \mathrm{~Hz}, 3 \mathrm{H}), 1.34-1.24(\mathrm{~m}, 4 \mathrm{H}), 0.83(\mathrm{t}, J=7.2 \mathrm{~Hz}$, $3 \mathrm{H}) ;{ }^{13} \mathrm{C}$ NMR $\left(101 \mathrm{MHz}, \mathrm{CDCl}_{3}\right) \delta: 178.8,177.0,143.6,140.8,138.4$, 131.8, 129.1, 128.8, 128.24, 128.16, 127.3, 126.5, 126.0, 74.8, 69.7, 59.1, $47.1,44.9,40.0,36.0,30.5,22.9,22.2,13.9 ;$ FTIR $\left(v_{\max } / \mathrm{cm}^{-1}\right): 2954$, 2849, 1724, 1512, 1367, 1209, 1128, 71; MS (EI, $m / z): 441(90)$, 385(50), 328(40), 237(20), 207(30), 181(100), 145(90), 91(90), 45(35). HRMS (EI) $\mathrm{C}_{29} \mathrm{H}_{34} \mathrm{NO}_{4}[\mathrm{M}+\mathrm{H}]^{+}: 460.2482$ (calculated); 460.2482 (found)

\section{Acknowledgments}

The financial support of the Scientific and Technological Research Council of Turkey and Federal Ministry of Education and Research (Germany) via the Intensified Cooperation Program (201T092) is gratefully acknowledged. We thank the Mass Spectrometry Laboratory of Dortmund University of Technology and the Environmental Research Centre of Izmir Institute of Technology for 
HRMS analyses and GC-MS analyses of a part of samples.

\section{Appendix A. Supplementary data}

Supplementary data related to this article can be found at https://doi.org/10.1016/j.tet.2018.05.030.

\section{References}

1. For selected reviews, see: a) Hoffmann-Röder A, Krause N. Angew Chem Int Ed. 2004;43:1196-1216;

b). In: Krause N, Hashmi ASK, eds. Modern Allene Chemistry. vols 1 and 2 . Weinheim: Wiley-VCH; 2004;

c) Ma S. Chem Rev. 2005; 105:2829-2871;

d) Brasholz M, Reissig H-U, Zimmer R. Acc Chem Res. 2009;42:45-56;

e) Yu S, Ma S. Angew Chem Int Ed. 2012;51:3074-3112;

f) Lu T, Lu Z, Ma Z-X, Zhang Y, Hsung RP. Chem Rev. 2013;113:4862-4904;

g) Zimmer R, Reissig H-U. Chem Soc Rev. 2014;43:2888-2903;

h) Pulis AP, Yeung K, Procter DJ. Chem Sci. 2017;8:5240-5247.

2. For reviews on the synthesis of allenes, see: a) Neff RK, Frantz DE. ACS Catal. 2014;4:519-528;

b) Yu S, Ma S. Chem Commun. 2011:5384-5418;

c) Ogasawara M. Tetrahedron Asymmetry, 2009:20:259-384

d) Brummond KM, DeForrest JE. Synthesis. 2007:795-818;

e) Krause N, Hoffmann-Röder A. Tetrahedron. 2004;60:11671-11694.

3. For selected recent examples on the synthesis of allenes, see: a) Yoshida M, Ueda H, Ihara M. Tetrahedron Lett. 2005;46:6705;

b) Molander GA, Sommers EM, Baker SR. J Org Chem. 2006;71:1563-1568;

c) Miura T, Shimada M, Ku S-Y, Tamai T, Murakami M. Angew Chem Int Ed. 2007:46:7101-7103;

d) Hayashi S, Hirano K, Yorimitsu H, Oshima K. J Am Chem Soc. 2008;130: 5048-5049;

e) Shu W, Jia G, Ma S. Org Lett. 2009;11:117-120;

f) Nishimura T, Makino H, Nagaosa M, Hayashi T. J Am Chem Soc. 2010;132: 12865-12867;

g) Yu S, Ma S. Chem Commun. 2011;47:5384-5418;

h) Wang Y, Zhang W, Ma S. J Am Chem Soc. 2013;135:11517-11520;

i) Hashimoto T, Sakata K, Tamakuni F, Dutton MJ, Maruoka K. Nat Chem. 2013;5:240-244; j) Ye J, Ma S. Org Chem Front. 2014;1:1210-1224;

k) Wu S, Huang X, Wu W, Li P, Fu C, Ma S. Nat Commun. 2015;6:1-8.

4. For selected reviews, see: (a) Ma S. Acc Chem Res. 2009;42:1679;

b) López F, Mascareñas J. Chem Eur J. 2011;17:418-428;

c) Alcaide B, Almendros P. Adv Synth Catal. 2011;353:2561-2576;

d) Lu T, Lu Z, Ma Z-X, Zhang Y, Hsung RP. Chem Rev. 2013;113:4862-4904;

e) Alcaide B, Almendros P. Acc Chem Res. 2014;47:939-952;

f) Ye J, Ma S. Acc Chem Res. 2014;47:989-1000;

g) Alcaide B, Almendros P. Chem Soc Rev. 2014;43:2879-3206.

5. a) Akpınar GE, Kuș M, Üçüncü M, Karakuș E, Artok L. Org Lett. 2011;13: 748-751;

b) Üçüncü M, Karakuş E, Kuş M, et al. J Org Chem. 2011;76:5959-5971;

c) Karagöz ES, Kus M, Akpinar GE, Artok L. J Org Chem. 2014;79:9222-9230;

d) Kuş M, Artok L, Aygün M. J Org Chem. 2015;80:5494-5506.

6. We have also disclosed recently that enyne acetates or enyne oxiranes react with Grignard reagents in the presence of an iron compound to provide vinylallenes: Aytaç IA, Karatavuk AO, Kuș M, Ziyanak F, Artok L Asian J Org Chem. 2017;6:1415-1420.

7. Moriya T, Miyaura N, Suzuki A. Synlett. 1994:149-151.

8. a) Hirai A, Yu X-Q. Tonooka T, Miyashita M. Chem Commun. 2003:2482-2483; b) Yu X-Q, Hirai A, Miyashita M. Chem Lett. 2004;33:764-765.

9. Wu K-M, Midland MM, Okamura WH. J Org Chem. 1990;55:4381-4392.

10. In an our previous report (ref. 5b), the transmetalation step was proposed to occur from the $\sigma$-allenylpalladium complex. However, the fact that the allylic substitution product $\mathbf{4 b f}$ formed as the major product when the coupling reaction was performed by using the bulky organoboron 1 ( Table 3 , entry 5 ) implies that $\pi$-allylpalladium intermediate $(\mathbf{A})$ should have undergone transmetalation before the migration of palladium.

11. a) Elsevier CJ, Kleijn H, Ruitenberg K, Vermeer P. J Chem Soc Chem Commun 1983:1529-1530;

b) Elsevier CJ, Kleijn H, Boersma J, Vermeer P. Organometallics. 1986;5: 716-720;

c) Wouters JMA, Klein RA, Elsevier CJ. Organometallics. 1994;13:4586-4593; d) Tsutsumi K, Ogoshi S, Kakiuchi K, Nishiguchi S, Kurosawa H. Inorg Chim Acta. 1999;296:37-44.

12. Enantioenriched allenes have been shown to undergo racemization in the presence of Pd/phosphine combination: a) Mikami K, Yoshida A. Angew Chem Int Ed Engl. 1997;36:858-860;

b) Ogoshi S, Nishida T, Shinagawa T, Kurosawa H. J Am Chem Soc. 2001;123: 7164-7165.

13. Corey EJ, Chaykovsky M. J Am Chem Soc. 1965;87:1353-1364. 